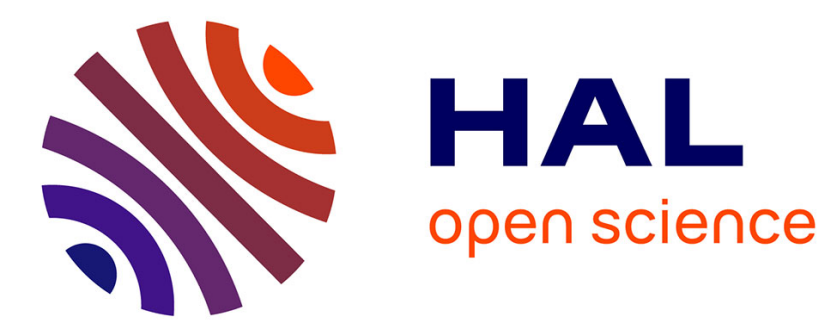

\title{
Micropolar Shells as Two-dimensional Generalized Continua Models
}

\author{
Holm Altenbach, Victor A. Eremeyev, Leonid P. Lebedev
}

\section{To cite this version:}

Holm Altenbach, Victor A. Eremeyev, Leonid P. Lebedev. Micropolar Shells as Two-dimensional Generalized Continua Models. Advanced Structured Materials Volume 7. Mechanics of Generalized Continua, Springer, pp.23-55, 2011, Volume 7. hal-00824010

\section{HAL Id: hal-00824010 https://hal.science/hal-00824010}

Submitted on 20 May 2013

HAL is a multi-disciplinary open access archive for the deposit and dissemination of scientific research documents, whether they are published or not. The documents may come from teaching and research institutions in France or abroad, or from public or private research centers.
L'archive ouverte pluridisciplinaire HAL, est destinée au dépôt et à la diffusion de documents scientifiques de niveau recherche, publiés ou non, émanant des établissements d'enseignement et de recherche français ou étrangers, des laboratoires publics ou privés. 


\title{
Micropolar Shells as Two-dimensional Generalized Continua Models
}

\author{
Holm Altenbach, Victor A. Eremeyev, and Leonid P. Lebedev
}

\begin{abstract}
Using the direct approach the basic relations of the nonlinear micropolar shell theory are considered. Within the framework of this theory the shell can be considered as a deformable surface with attached three unit orthogonal vectors, so-called directors. In other words the micropolar shell is a two-dimensional (2D) Cosserat continuum or micropolar continuum. Each point of the micropolar shell has three translational and three rotational degrees of freedom as in the rigid body dynamics. In this theory the rotations are kinematically independent on translations. The interaction between of any two parts of the shell is described by the forces and moments only. So at the shell boundary six boundary conditions have to be given. In contrast to Kirchhoff-Love or Reissner's models of shells the drilling moment acting on the shell surface can be taken into account.

In the paper we derive the equilibrium equations of the shell theory using the principle of virtual work. The strain measures are introduced on the base of the principle of frame indifference. The boundary-value static and dynamic problems are formulated in Lagrangian and Eulerian coordinates. In addition, some variational principles are presented. For the general constitutive equations we formulate some constitutive restrictions, for example, the Coleman-Noll inequality, the Hadamard inequality, etc. Finally, we discuss the equilibrium of shells made of materials un-
\end{abstract}

Holm Altenbach

Martin-Luther-Universität Halle-Wittenberg, Kurt-Mothes-Str. 1, 06099 Halle (Saale), Germany e-mail: holm.altenbach@iw.uni-halle.de

Victor A. Eremeyev

Martin-Luther-Universität Halle-Wittenberg, Kurt-Mothes-Str. 1, 06099 Halle (Saale), Germany; South Scientific Center of RASci \& South Federal University, Milchakova St. 8a, 344090 Rostov on Don, Russia

e-mail: eremeyev.victor@gmail.com;victor.eremeyev@iw.uni-halle.de

Leonid P. Lebedev

Universidad Nacional de Colombia, Cr. 45, \# 2685, Bogotá D.C., Colombia

e-mail: 1lebedev@unal.edu.co 
dergoing phase transformations, such as martensitic transformations, and formulate the compatibility conditions on the phase interface.

Key words: Micropolar shells. 6-parametric theory of shells. Variational principles. Constitutive inequalities. Coleman-Noll inequality. Hadamard inequality. Phase transformations.

\subsection{Introduction}

The Mechanics of Generalized Continua has long history of development. Since the centurial book of Cosserat brothers [13] in the literature there are known various generalizations of the classical or Cauchy continuum which are summarized in many books and papers, see, for example, the books by Eringen $[25,26]$, Nowacki [47], and the recent proceedings [45], see also the historical review by Maugin [44] in this book. Using the direct approach, Ericksen and Truesdell [24] extended the Cosserat model to construction of the nonlinear mechanics of rods and shells, i.e. to one-dimensional and two-dimensional media. Since [24] the generalized models of shells and plates are extensively discussed in the literature, see the recent review [5].

Below we consider the model of a micropolar shell as the example of the generalized 2D continuum. Indeed, a micropolar shell is a two-dimensional analogue of the three-dimensional (3D) micropolar continuum, i.e. a micropolar shell is a deformable directed surface each particle of which has six degrees of freedom as rigid bodies. The kinematics of the micropolar shell is described by two fields. The first field is the position vector of the base surface of the shell while the second one is the proper orthogonal tensor describing the rotation of the shell cross-section. In contrast to Kirchhoff-Love and Mindlin-Reissner type theories of plates and shells the boundary-value problem of a micropolar shell consists of 6 scalar equations and 6 boundary conditions. Within the micropolar shell theory the so-called drilling moment can be taken into account.

Let us note that the basic equations of the micropolar shell models presented in $[16,22,23,60]$ using the direct approach coincide with the general nonlinear theory of shells initiated by Reissner [55] and presented by Libai and Simmonds [38, 39], Pietraszkiewicz [51], and Chróścielewski et al. [10], which is also named 6-parametric theory of shells.

The paper is organized as follows. In Sect. 2.2 we recall the basic equations. We derive the equilibrium equations from the principle of virtual work. Various statements of the nonlinear boundary-value problem are given and few variational principles are formulated. The case of small deformations is also considered. Following [22] in Sect. 2.3 we present in details some inequalities such as the Coleman-Noll inequality, the Hadamard inequality, strong and ordinary ellipticity conditions, etc. These inequalities can be regarded as the constitutive restrictions, i.e. the restrictions for the constitutive equations of an elastic shell. In Sect. 2.4 we discuss the compatibility conditions on the phase interface in shells. 
Further we use the direct tensorial notations, see for example [36, 42]. Vectors are denoted by semibold normal font like $\mathbf{A}$. Tensors are denoted by semibold sans serif upright font like $\mathbf{A}$. Functionals are denoted by calligraphic letters like $\mathcal{A}$. Greek indices take values 1 and 2, while Latin indices are arbitrary.

\subsection{Basic Relations of Micropolar Shell Theory}

In this section we use the so-called direct approach to the formulation of the basic equations of micropolar shell theory. The advantage of the latter approach is discussed in many papers, see for example [24]. Within framework of the direct approach, an elastic micropolar shell is a two-dimensional analogue of the Cosserat continuum, i.e. a micropolar shell is a material surface each particle of which has six degrees of freedom of the rigid body. Further we will use the notations [16, 22, 23].

\subsubsection{Kinematics of a Micropolar Shell}

Let $\sigma$ be a base surface of the micropolar shell in the reference configuration (for example, in an undeformed state), $q^{\alpha}(\alpha=1,2)$ be Gaussian coordinates on $\sigma$, and $\mathbf{r}\left(q^{1}, q^{2}\right)$ be a position vector of $\sigma$, see Fig. 2.1. In the actual (deformed) configuration the surface is denoted by $\Sigma$, and the position of its material points (infinitesimal point-bodies) is given by the vector $\mathbf{R}\left(q^{1}, q^{2}\right)$. The orientation of the pointbodies is described by the so-called microrotation tensor (or turn-tensor) $\mathbf{Q}\left(q^{1}, q^{2}\right)$, which is the proper orthogonal tensor. If we introduce three orthonormal vectors $\mathbf{d}_{k}$ $(k=1,2,3)$, which describe the orientation in the reference configuration, and three

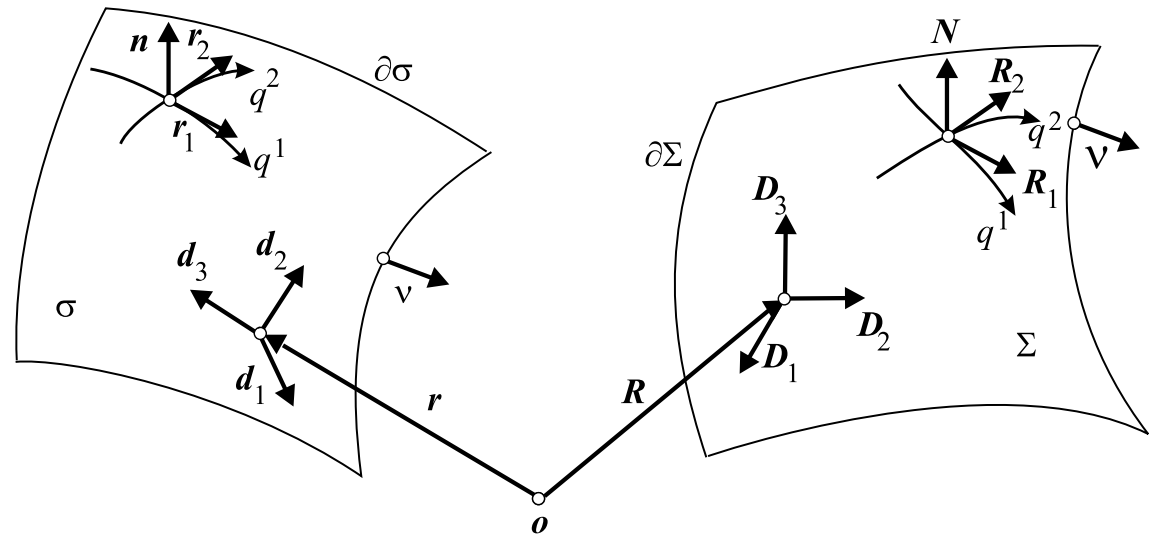

Fig. 2.1 Kinematics of a micropolar shell, reference configuration (on the left) and actual one (on the right) 
orthonormal vectors $\mathbf{D}_{k}$, which determine the orientation in the actual configuration, then the tensor $\mathbf{Q}$ is given by $\mathbf{Q}=\mathbf{d}_{k} \otimes \mathbf{D}_{k}$. Thus, the micropolar shell is described by two kinematically independent fields

$$
\mathbf{R}=\mathbf{R}\left(q^{\alpha}\right), \quad \mathbf{Q}=\mathbf{Q}\left(q^{\alpha}\right) .
$$

For the micropolar shell made of an elastic material there should exist a strain energy density $W$. By using the principle of local action $[57,58]$ the constitutive equation for the function $W$ is given by the formula $[16,22,23]$

$$
W=W(\mathbf{R}, \nabla \mathbf{R}, \mathbf{Q}, \nabla \mathbf{Q}),
$$

where

$$
\nabla \Psi \triangleq \mathbf{r}^{\alpha} \otimes \frac{\partial \Psi}{\partial q^{\alpha}}, \quad \mathbf{r}^{\alpha} \cdot \mathbf{r}_{\beta}=\delta_{\beta}^{\alpha}, \quad \mathbf{r}^{\alpha} \cdot \mathbf{n}=0, \quad \mathbf{r}_{\beta}=\frac{\partial \mathbf{r}}{\partial q^{\beta}} .
$$

Here the vectors $\mathbf{r}_{\beta}$ and $\mathbf{r}^{\alpha}$ denote the natural and reciprocal bases on $\sigma, \mathbf{n}$ is the unit normal to $\sigma, \delta_{\beta}^{\alpha}$ is the Kronecker symbol, $\nabla$ is the surface nabla operator on $\sigma$, and $\Psi$ is an arbitrary differentiable tensor field given on $\sigma$.

From the principle of material frame-indifference we can find that $W$ depends on two Cosserat-type strain measures $\mathbf{E}$ and $\mathbf{K}$ only

$$
\begin{gathered}
W=W(\mathbf{E}, \mathbf{K}), \\
\mathbf{E}=\mathbf{F} \cdot \mathbf{Q}^{T}, \quad \mathbf{K}=\frac{1}{2} \mathbf{r}^{\alpha} \otimes\left(\frac{\partial \mathbf{Q}}{\partial q^{\alpha}} \cdot \mathbf{Q}^{T}\right)_{\times},
\end{gathered}
$$

where $\mathbf{F}=\nabla \mathbf{R}$ is the surface deformation gradient, $\mathbf{T}_{\times}$is the vectorial invariant of a second-order tensor $\mathbf{T}$ defined by

$$
\mathbf{T}_{\times}=\left(T^{m n} \mathbf{R}_{m} \otimes \mathbf{R}_{n}\right)_{\times}=T^{m n} \mathbf{R}_{m} \times \mathbf{R}_{n}
$$

for any base $\mathbf{R}_{m}$, see e.g. [36], $\times$ is the vector product.

A proper orthogonal tensor describes rotation about an arbitrary axis. It can be represented by Gibbs' formula

$$
\mathbf{H}=(\mathbf{I}-\mathbf{e} \otimes \mathbf{e}) \cos \chi+\mathbf{e} \otimes \mathbf{e}-\mathbf{e} \times \mathbf{I} \sin \chi,
$$

where $\chi$ and $\mathbf{e}$ are the angle of rotation about the axis with the unit vector $\mathbf{e}$, and $\mathbf{I}$ is the $3 \mathrm{D}$ unit tensor, respectively. Introducing the vector $\boldsymbol{\theta}=2 \mathbf{e} \tan \chi / 2$ and using the formulae

$$
\cos \chi=\frac{1-\tan ^{2} \chi / 2}{1+\tan ^{2} \chi / 2}, \quad \sin \chi=\frac{2 \tan \chi / 2}{1+\tan ^{2} \chi / 2}
$$

we obtain the representation of $\mathbf{Q}$ in the form which does not contain trigonometric functions

$$
\mathbf{Q}=\frac{1}{\left(4+\theta^{2}\right)}\left[\left(4-\theta^{2}\right) \mathbf{I}+2 \theta \otimes \theta-4 \mathbf{I} \times \theta\right], \quad \theta^{2}=\theta \cdot \theta
$$


In the rigid body kinematics the vector $\boldsymbol{\theta}$ is called Rodrigues' finite rotation vector, cf. [41]. In the theory of Cosserat-type shells we will call it the microrotation vector. Other known vectorial parameterizations of an orthogonal tensor are summarized in [53]. From Eq. (2.4), for a given proper orthogonal tensor $\mathbf{Q}$ we find uniquely the vector $\boldsymbol{\theta}$

$$
\boldsymbol{\theta}=2(1+\operatorname{tr} \mathbf{Q})^{-1} \mathbf{Q}_{\times}
$$

Using the finite rotation vector we can express $\mathbf{K}$ as follows

$$
\mathbf{K}=\mathbf{r}^{\alpha} \otimes \mathbf{L}_{\alpha}=\frac{4}{4+\theta^{2}} \nabla \boldsymbol{\theta} \cdot\left(\mathbf{I}+\frac{1}{2} \mathbf{I} \times \boldsymbol{\theta}\right) .
$$

The strain measures $\mathbf{E}$ and $\mathbf{K}$ are the two-dimensional analogues of the strain measures used in $3 \mathrm{D}$ Cosserat continuum $[52,53]$.

\subsubsection{Principle of Virtual Work and Boundary-value Problems Statements}

The Lagrangian equilibrium equations of the micropolar shell can be derived from the principle of virtual work

$$
\delta \iint_{\sigma} W d \sigma=\delta^{\prime} A
$$

where

$$
\begin{gathered}
\delta^{\prime} A=\iint_{\sigma}\left(\mathbf{f} \cdot \delta \mathbf{R}+\mathbf{c} \cdot \delta^{\prime} \psi\right) d \sigma+\int_{\omega_{2}} \varphi \cdot \delta \mathbf{R} d s+\int_{\omega_{4}} \gamma \cdot \delta^{\prime} \psi d s \\
\mathbf{I} \times \delta^{\prime} \psi=-\mathbf{Q}^{T} \cdot \delta \mathbf{Q} .
\end{gathered}
$$

In Eq. (2.7), $\delta$ is the symbol of variation, $\delta^{\prime} \psi$ is the virtual rotation vector, $\mathbf{f}$ is the surface force density distributed on $\sigma, \mathbf{c}$ is the surface couple density distributed on $\sigma, \varphi$ and $\gamma$ are linear densities of forces and couples distributed along corresponding parts of the shell boundary $\omega$, respectively.

Using the formulae [23]

$$
\begin{gathered}
\delta W=\frac{\partial W}{\partial \mathbf{E}} \bullet \delta \mathbf{E}+\frac{\partial W}{\partial \mathbf{K}} \bullet \delta \mathbf{K}, \\
\delta \mathbf{E}=(\nabla \delta \mathbf{R}) \cdot \mathbf{Q}^{T}+\mathbf{F} \cdot \delta \mathbf{Q}^{T}, \quad \delta \mathbf{K}=\left(\nabla \delta^{\prime} \boldsymbol{\psi}\right) \cdot \mathbf{Q}^{T}, \\
\delta^{\prime} \boldsymbol{\psi}=\frac{4}{4+\theta^{2}}\left(\delta \boldsymbol{\theta}+\frac{1}{2} \boldsymbol{\theta} \times \delta \theta\right),
\end{gathered}
$$


where $\mathbf{X} \bullet \mathbf{Y}=\mathbf{X} \cdot \mathbf{Y}^{T}$ for any tensors $\mathbf{X}$ and $\mathbf{Y}$ of the second-order, see [36], from Eq. (2.7) we obtain the Lagrangian shell equations

$$
\begin{aligned}
\boldsymbol{\nabla} \cdot \mathbf{D}+\mathbf{f}=\mathbf{0}, \quad \boldsymbol{\nabla} \cdot \mathbf{G}+\left[\mathbf{F}^{T} \cdot \mathbf{D}\right]_{\times}+\mathbf{c}=\mathbf{0}, \\
\mathbf{D}=\mathbf{P}_{1} \cdot \mathbf{Q}, \quad \mathbf{G}=\mathbf{P}_{2} \cdot \mathbf{Q}, \quad \mathbf{P}_{1}=\frac{\partial W}{\partial \mathbf{E}}, \quad \mathbf{P}_{2}=\frac{\partial W}{\partial \mathbf{K}}, \\
\omega_{1}: \mathbf{R}=\boldsymbol{\rho}(s), \\
\omega_{2}: \boldsymbol{v} \cdot \mathbf{D}=\boldsymbol{\varphi}(s), \\
\omega_{3}: \mathbf{Q}=\mathbf{h}(s), \mathbf{h} \cdot \mathbf{h}^{T}=\mathbf{I}, \\
\omega_{4}: v \cdot \mathbf{G}=\gamma(s) .
\end{aligned}
$$

Here $\rho(s), \mathbf{h}(s)$ are given vector functions, and $v$ is the external unit normal to the boundary curve $\omega(v \cdot \mathbf{n}=0)$. Equations (2.8) are the equilibrium equations for the linear momentum and angular momentum of any shell part. The tensors $\mathbf{D}$ and $\mathbf{G}$ are the surface stress and couple stress tensors of the $1^{\text {st }}$ Piola-Kirchhoff type, and the corresponding stress measures $\mathbf{P}_{1}$ and $\mathbf{P}_{2}$ in Eqs (2.8) are the referential stress tensors, respectively. The strain measures $\mathbf{E}$ and $\mathbf{K}$ are work-conjugate to the stress measures $\mathbf{D}$ and $\mathbf{G}$. The boundary $\omega$ of $\sigma$ is divided into two parts $\omega=\omega_{1} \cup \omega_{2}=$ $\omega_{3} \cup \omega_{4}$. The following relations are valid

$$
\mathbf{n} \cdot \mathbf{D}=\mathbf{n} \cdot \mathbf{G}=\mathbf{n} \cdot \mathbf{P}_{1}=\mathbf{n} \cdot \mathbf{P}_{2}=\mathbf{0} .
$$

The equilibrium equations (2.8) may be transformed to the Eulerian form

$$
\nabla_{\Sigma} \cdot \mathbf{T}+J^{-1} \mathbf{f}=\mathbf{0}, \quad \nabla_{\Sigma} \cdot \mathbf{M}+\mathbf{T}_{\times}+J^{-1} \mathbf{c}=\mathbf{0},
$$

where

$$
\begin{gathered}
\nabla_{\Sigma} \cdot \Psi \triangleq \mathbf{R}^{\alpha} \cdot \frac{\partial \Psi}{\partial q^{\alpha}}, \quad \mathbf{R}^{\alpha} \cdot \mathbf{R}_{\beta}=\delta_{\beta}^{\alpha}, \mathbf{R}^{\alpha} \cdot \mathbf{N}=0, \quad \mathbf{R}_{\beta}=\frac{\partial \mathbf{R}}{\partial q^{\beta}}, \\
\mathbf{T}=J^{-1} \mathbf{F}^{T} \cdot \mathbf{D}, \quad \mathbf{M}=J^{-1} \mathbf{F}^{T} \cdot \mathbf{G}, \\
J=\sqrt{\frac{1}{2}\left\{\left[\operatorname{tr}\left(\mathbf{F} \cdot \mathbf{F}^{T}\right)\right]^{2}-\operatorname{tr}\left[\left(\mathbf{F} \cdot \mathbf{F}^{T}\right)^{2}\right]\right\}} .
\end{gathered}
$$

Here $\mathbf{T}$ and $\mathbf{M}$ are the Cauchy-type surface stress and couple stress tensors, $\nabla_{\Sigma}$ is the surface nabla operator on $\Sigma$ associated with $\nabla$ by the formula $\nabla=\mathbf{F} \cdot \nabla_{\Sigma}$.

The equations of motion of the micropolar shell are given by the relations (see, for example, $[4,10,23,38,39,59])$

$$
\begin{gathered}
\nabla \cdot \mathbf{D}+\mathbf{f}=\rho \frac{\mathrm{d} \mathbf{K}_{1}}{\mathrm{~d} t}, \\
\nabla \cdot \mathbf{G}+\left[\mathbf{F}^{T} \cdot \mathbf{D}\right]_{\times}+\mathbf{m}=\rho\left(\frac{\mathrm{d} \mathbf{K}_{2}}{\mathrm{~d} t}+\mathbf{v} \times \boldsymbol{\Theta}_{1}^{T} \cdot \boldsymbol{\omega}\right),
\end{gathered}
$$

with 


$$
\begin{gathered}
K(\mathbf{v}, \boldsymbol{\omega})=\frac{1}{2} \mathbf{v} \cdot \mathbf{v}+\boldsymbol{\omega} \cdot \Theta_{1} \cdot \mathbf{v}+\frac{1}{2} \boldsymbol{\omega} \cdot \Theta_{2} \cdot \omega \\
\mathbf{K}_{1} \triangleq \frac{\partial K}{\partial \mathbf{v}}=\mathbf{v}+\Theta_{1}^{T} \cdot \omega, \quad \mathbf{K}_{2} \triangleq \frac{\partial K}{\partial \boldsymbol{\omega}}=\Theta_{1} \cdot \mathbf{v}+\Theta_{2} \cdot \omega
\end{gathered}
$$

where

$$
\mathbf{v}=\frac{d \mathbf{R}}{d t}, \quad \boldsymbol{\omega}=\frac{1}{2}\left(\mathbf{H}^{T} \cdot \frac{d \mathbf{Q}}{d t}\right)_{\times}
$$

are the linear and angular velocities, respectively, $\rho$ is the surface mass density in the reference configuration, $\rho K$ is the surface density of the kinetic energy, and $\rho \boldsymbol{\Theta}_{1}$, $\rho \boldsymbol{\Theta}_{2}$ are the rotatory inertia tensors $\left(\boldsymbol{\Theta}_{2}^{T}=\boldsymbol{\Theta}_{2}\right)$. For the dynamic problem (2.14), the initial conditions are given by

$$
\left.\mathbf{R}\right|_{t=0}=\mathbf{R}^{\circ},\left.\quad \mathbf{v}\right|_{t=0}=\mathbf{v}^{\circ},\left.\quad \mathbf{Q}\right|_{t=0}=\mathbf{Q}^{\circ},\left.\quad \boldsymbol{\omega}\right|_{t=0}=\omega^{\circ},
$$

where $\mathbf{R}^{\circ}, \mathbf{v}^{\circ}, \mathbf{Q}^{\circ}, \boldsymbol{\omega}^{\circ}$ are given initial values.

Under some conditions the equilibrium problem of a micropolar shell can be transformed to the system of equations with respect to the strain measures

$$
\begin{gathered}
\nabla \cdot \mathbf{P}_{1}-\left(\mathbf{P}_{1}^{T} \cdot \mathbf{K}\right)_{\times}+\mathbf{f}^{*}=\mathbf{0} ; \\
\nabla \cdot \mathbf{P}_{2}-\left(\mathbf{P}_{2}^{T} \cdot \mathbf{K}+\mathbf{P}_{1}^{T} \cdot \mathbf{E}\right)_{\times}+\mathbf{c}^{*}=\mathbf{0}, \\
\omega_{2}: \boldsymbol{v} \cdot \mathbf{P}_{1}=\boldsymbol{\varphi}^{*}, \quad \omega_{4}: \boldsymbol{v} \cdot \mathbf{P}_{2}=\boldsymbol{\gamma}^{*}, \\
\mathbf{f}^{*} \triangleq \mathbf{f} \cdot \mathbf{Q}^{T}, \mathbf{c}^{*} \triangleq \mathbf{c} \cdot \mathbf{Q}^{T}, \quad \boldsymbol{\varphi}^{*} \triangleq \boldsymbol{\varphi} \cdot \mathbf{Q}^{T}, \quad \gamma^{*} \triangleq \boldsymbol{\gamma} \cdot \mathbf{Q}^{T} .
\end{gathered}
$$

Let the vectors $\mathbf{f}^{*}, \mathbf{c}^{*}, \varphi^{*}, \gamma^{*}$ be given as functions of the coordinates $q^{1}, q^{2}$. From the physical point of view it means that the shell is loaded by tracking forces and couples. Then Eqs (2.15)-(2.17) depend on $\mathbf{E}, \mathbf{K}$ as the only independent fields.

\subsubsection{On the Constitutive Equations of Micropolar Shells}

For an elastic shell the constitutive equations consist of the surface strain energy density as the function of two strain measures. An example of the constitutive equation is the model of physically linear isotropic shell $[10,18,23]$, the energy of which is given by the quadratic form

$$
\begin{aligned}
2 W & =\alpha_{1} \operatorname{tr}^{2} \mathbf{E}_{\|}+\alpha_{2} \operatorname{tr} \mathbf{E}_{\|}^{2}+\alpha_{3} \operatorname{tr}\left(\mathbf{E}_{\|} \cdot \mathbf{E}_{\|}^{T}\right)+\alpha_{4} \mathbf{n} \cdot \mathbf{E}^{T} \cdot \mathbf{E} \cdot \mathbf{n} \\
& +\beta_{1} \operatorname{tr}^{2} \mathbf{K}_{\|}+\beta_{2} \operatorname{tr} \mathbf{K}_{\|}^{2}+\beta_{3} \operatorname{tr}\left(\mathbf{K}_{\|} \cdot \mathbf{K}_{\|}^{T}\right)+\beta_{4} \mathbf{n} \cdot \mathbf{K}^{T} \cdot \mathbf{K} \cdot \mathbf{n}, \\
\mathbf{E}_{\|} & \triangleq \mathbf{E} \cdot \mathbf{A}, \quad \mathbf{K}_{\|} \triangleq \mathbf{K} \cdot \mathbf{A} .
\end{aligned}
$$

In Eq. (2.18) there are absent the terms that are bilinear in $\mathbf{E}$ and $\mathbf{K}$. It is a consequence of the fact that the bending measure $\mathbf{K}$ is a pseudo-tensor that changes the 
sign of the value when we apply the inversion of the space. Note that the constitutive equations contain 8 parameters $\alpha_{k}, \beta_{k}(k=1,2,3,4)$.

In [10] the following relations for the elastic moduli appearing in Eq. (2.18) are used

$$
\begin{aligned}
& \alpha_{1}=C v, \quad \alpha_{2}=0, \quad \alpha_{3}=C(1-v), \quad \alpha_{4}=\alpha_{s} C(1-v), \\
& \beta_{1}=D v, \quad \beta_{2}=0, \quad \beta_{3}=D(1-v), \quad \beta_{4}=\alpha_{t} D(1-v), \\
& C=\frac{E h}{1-v^{2}}, \quad D=\frac{E h^{3}}{12\left(1-v^{2}\right)},
\end{aligned}
$$

where $E$ and $v$ are the Young's modulus and the Poisson's ratio of the bulk material, respectively, $\alpha_{s}$ and $\alpha_{t}$ are dimensionless shear correction factors, while $h$ is the shell thickness. $\alpha_{s}$ is the shear correction factor introduced in the plate theory by Reissner $\left(\alpha_{s}=5 / 6\right)$ or Mindlin $\left(\alpha_{s}=\pi^{2} / 12\right)$. The parameter $\alpha_{t}$ plays the same role for the couple stresses. The value $\alpha_{t}=0.7$ was proposed by Pietraszkiewicz $[48,49]$, see also [11]. In $[10,11,12]$ the influence of $\alpha_{s}$ and $\alpha_{t}$ on the solution is investigated numerically for several boundary-value problems.

\subsubsection{Compatibility Conditions}

Let us consider how to determine the position vector $\mathbf{R}\left(q^{1}, q^{2}\right)$ of $\Sigma$ from the surface strain $\mathbf{E}$ and micro-rotation $\mathbf{Q}$, which are assumed to be given as continuously differentiable functions on $\sigma$. By using the equation $\mathbf{F}=\mathbf{E} \cdot \mathbf{Q}$ the problem is reduced to

$$
\nabla \mathbf{R}=\mathbf{F} .
$$

The necessary and sufficient condition for solvability of Eq. (2.20) is given by the relation

$$
\boldsymbol{\nabla} \cdot(\mathbf{e} \cdot \mathbf{F})=\mathbf{0}, \quad \mathbf{e} \triangleq-\mathbf{I} \times \mathbf{n},
$$

which we call the compatibility condition for the distortion tensor $\mathbf{F}$. Here $\mathbf{e}$ is the skew-symmetric discriminant tensor on the surface $\sigma$. For a simply-connected region $\sigma$, if the condition (2.21) is satisfied, the vector field $\mathbf{R}$ may be deduced from Eq. (2.20) only up to an additive vector.

Let us consider a more complex problem of determination of both the translations and rotations of the micropolar shell from the given fields of $\mathbf{E}$ and $\mathbf{K}$. At first, let us deduce the field $\mathbf{Q}\left(q^{1}, q^{2}\right)$ by using the system of equations following from definition (2.2) of $\mathbf{K}$

$$
\frac{\partial \mathbf{Q}}{\partial q^{\alpha}}=-\mathbf{K}_{\alpha} \times \mathbf{Q}, \quad \mathbf{K}_{\alpha} \triangleq \mathbf{r}_{\alpha} \cdot \mathbf{K} .
$$

The integrability conditions for the system (2.22) are given by the relation 


$$
\frac{\partial \mathbf{K}_{\alpha}}{\partial q^{\beta}}-\frac{\partial \mathbf{K}_{\beta}}{\partial q^{\alpha}}=\mathbf{K}_{\alpha} \times \mathbf{K}_{\beta} \quad(\alpha, \beta=1,2)
$$

Equations (2.23) are obtained in $[38,49,50]$ as the conditions for the existence of the rotation field of the shell. They may be written in the following coordinate-free form

$$
\begin{gathered}
\nabla \cdot(\mathbf{e} \cdot \mathbf{K})+\mathbf{K}^{\perp} \cdot \mathbf{n}=\mathbf{0}, \\
\mathbf{K}^{\perp} \triangleq \frac{1}{2}\left(\mathbf{K}_{\alpha} \times \mathbf{K}_{\beta}\right) \otimes\left(\mathbf{r}^{\alpha} \times \mathbf{r}^{\beta}\right)=\mathbf{K}^{2}-\mathbf{K} \operatorname{tr} \mathbf{K}+\frac{1}{2}\left(\operatorname{tr}^{2} \mathbf{K}-\operatorname{tr} \mathbf{K}^{2}\right) \mathbf{I} .
\end{gathered}
$$

Using $\mathbf{F}=\mathbf{E} \cdot \mathbf{Q}$ and Eqs (2.2) the compatibility condition (2.21) may be written in the form

$$
\nabla \cdot(\mathbf{e} \cdot \mathbf{E})+\left(\mathbf{E}^{T} \cdot \mathbf{e} \cdot \mathbf{K}\right)_{\times}=\mathbf{0} .
$$

Two coordinate-free vector equations (2.24), (2.25) are the compatibility conditions for the nonlinear micropolar shell. These conditions and the system of equations (2.15)-(2.17) form the complete boundary-value problem for statics of micropolar shells expressed entirely in terms of the surface strain measures $\mathbf{E}$ and $\mathbf{K}$.

\subsubsection{Variational Statements}

The presented above static and dynamic problems have corresponding variational statements. Two of them for statics and one for dynamics are presented below.

\subsubsection{Lagrange Principle}

Let us assume that the external forces and couples are conservative. In the Lagrangetype variational principle $\delta \mathcal{E}_{1}=0$ the functional $\mathcal{E}_{1}[\mathbf{R}, \mathbf{Q}]$ is used, where

$$
\mathcal{E}_{1}[\mathbf{R}, \mathbf{Q}]=\iint_{\sigma} W \mathrm{~d} \sigma-\mathcal{A}[\mathbf{R}, \mathbf{Q}],
$$

and $\mathcal{A}$ is the potential of the external loads.

Here the deformation, i.e. the translations and the rotations, have to satisfy the boundary conditions $(2.10)_{1}$ and $(2.10)_{3}$ on $\omega_{1}$ and $\omega_{3}$, respectively. The stationarity of $\varepsilon_{1}$ is equivalent to the equilibrium equations (2.8), (2.9) and the boundary conditions $(2.10)_{2}$ and $(2.10)_{4}$ on $\omega_{2}$ and $\omega_{4}$.

\subsubsection{Hu-Washizu Principle}

For this principle the functional is given by 


$$
\begin{gathered}
\mathcal{E}_{2}\left[\mathbf{R}, \mathbf{Q}, \mathbf{E}, \mathbf{K}, \mathbf{D}, \mathbf{P}_{2}\right]=\iint_{\sigma}[W(\mathbf{E}, \mathbf{K})-\mathbf{D} \bullet(\mathbf{E} \cdot \mathbf{Q}-\nabla \mathbf{R}) \\
\left.-\mathbf{P}_{2} \bullet\left(\mathbf{K}-\frac{1}{2} \mathbf{r}^{\alpha} \otimes\left(\frac{\partial \mathbf{Q}}{\partial q^{\alpha}} \cdot \mathbf{Q}^{T}\right)_{\times}\right)\right] \mathrm{d} \sigma-\int_{\omega_{1}} \boldsymbol{v} \cdot \mathbf{D} \cdot(\mathbf{R}-\boldsymbol{\rho}) \mathrm{d} s-\mathcal{A}[\mathbf{R}, \mathbf{Q}] .
\end{gathered}
$$

From the condition $\delta \varepsilon_{2}=0$ the equilibrium equations (2.8) and (2.9), the constitutive equations, and the relations (2.2) are deduced. For this principle the natural boundary conditions are given by the relations $(2.10)_{1},(2.10)_{2}$ and $(2.10)_{4}$, respectively.

Several other variational statements are given in [23]. Mixed type variational functionals are constructed in [10]. They are used for the development of a family of finite elements with 6 degrees of freedom in each node. Then a number of nonlinear simulations of complex multifolded shell structures are performed.

\subsubsection{Hamilton-type Principle}

The kinetic energy of micropolar shells can be expressed as

$$
\mathcal{K}=\iint_{\sigma} \rho K(\mathbf{v}, \omega) \mathrm{d} \sigma, \quad K(\mathbf{v}, \boldsymbol{\omega})=\frac{1}{2} \mathbf{v} \cdot \mathbf{v}+\omega \cdot \Theta_{1} \cdot \mathbf{v}+\frac{1}{2} \omega \cdot \Theta_{2} \cdot \omega,
$$

where $\rho K$ is the surface density of the kinetic energy, $\rho \boldsymbol{\Theta}_{1}, \rho \Theta_{2}$ are the inertia tensors, $\boldsymbol{\Theta}_{2}^{T}=\boldsymbol{\Theta}_{2}$.

It is obvious that we should assume the kinetic energy to be a positive definite function that imposes some restriction on the form of the inertia tensors. By physical meaning, $\boldsymbol{\Theta}_{1}$ and $\boldsymbol{\Theta}_{2}$ have the following properties

$$
\boldsymbol{\Theta}_{1}=\mathbf{H}^{T} \cdot \boldsymbol{\Theta}_{1}^{\circ} \cdot \mathbf{H}, \quad \Theta_{2}=\mathbf{H}^{T} \cdot \Theta_{2}^{\circ} \cdot \mathbf{H}, \quad \frac{d \boldsymbol{\Theta}_{1}^{\circ}}{d t}=\frac{d \boldsymbol{\Theta}_{2}^{\circ}}{d t}=\mathbf{0} .
$$

The Hamilton principle is a variational principle in dynamics. In real motion, the functional

$$
\mathcal{E}_{3}[\mathbf{R}, \mathbf{H}]=\int_{t_{0}}^{t_{1}}\left(\mathcal{K}-\mathcal{E}_{1}\right) \mathrm{d} t
$$

takes a stationary value on the set of all possible shell motions that at the range $t_{0}$, $t_{1}$ take given values of the real motion values and satisfy the kinematic boundary values. In other words, its first variation on a real motion is zero. From condition $\varepsilon_{3}=0$ Eqs (2.14) can be established. 


\subsubsection{Linear Theory of Micropolar Shells}

Let us suppose the strains are small. Then we can simplify the equations of the shell theory significantly. In this geometrically linear case we do not differ Eulerian and Lagrangian descriptions. The difference of surfaces $\sigma$ and $\Sigma$ is infinitesimal. It is not necessary to distinguish operators $\nabla$ and $\nabla_{\Sigma}$ as well as earlier different types of stress tensors and couple stress tensors. Let us introduce the vector of infinitesimal displacements $\mathbf{u}$ and the vector of infinitesimal rotation $\vartheta$ such that there hold

$$
\mathbf{R} \approx \mathbf{r}+\mathbf{u}, \quad \mathbf{Q} \approx \mathbf{I}-\mathbf{I} \times \boldsymbol{\vartheta}
$$

In Eqs (2.31) the last formula follows from the representation of a proper orthogonal tensor through the finite rotation vector (2.4) if $|\boldsymbol{\theta}| \ll 1$.

Up to the linear addendum, the linear strain measure $\mathbf{E}$ and bending strain tensor can be expressed in terms of the linear strain tensor and linear bending strain tensor e and $\kappa$

$$
\mathbf{E} \approx \mathbf{I}+\mathbf{e}, \quad \mathbf{K} \approx \boldsymbol{\kappa}, \quad \mathbf{e}=\nabla \mathbf{u}+\mathbf{A} \times \vartheta, \quad \kappa=\nabla \vartheta .
$$

Here $\mathbf{A} \triangleq \mathbf{I}-\mathbf{n} \otimes \mathbf{n}$. The tensors $\mathbf{e}$ and $\kappa$ are applied in the linear theory of micropolar shells, cf. [59, 60]. Assuming Eq. (2.32) in the linear shell theory the stress tensors $\mathbf{D}, \mathbf{P}_{1}, \mathbf{T}$ and the couple tensors $\mathbf{G}, \mathbf{P}_{2}, \mathbf{M}$ coincide. In what follows we will denote the stress tensor by $\mathbf{T}$ and the couple stress tensor by $\mathbf{M}$.

The constitutive equations of an elastic shell can be represented through the function of specific strain energy $W=W(\mathbf{e}, \kappa)$ as it follows

$$
\mathbf{T}=\frac{\partial W}{\partial \mathbf{e}}, \quad \mathbf{M}=\frac{\partial W}{\partial \kappa} .
$$

In the linear theory the equilibrium equations take the form

$$
\nabla \cdot \mathbf{T}+\mathbf{f}=\mathbf{0}, \quad \nabla \cdot \mathbf{M}+\mathbf{T}_{\times}+\mathbf{c}=\mathbf{0},
$$

whereas the boundary conditions are transformed to

$$
\begin{aligned}
& \omega_{1}: \mathbf{u}=\mathbf{u}_{0}(s), \\
& \omega_{2}: v \cdot \mathbf{T}=\boldsymbol{\varphi}(s), \\
& \omega_{3}: \vartheta=\vartheta_{0}(s), \\
& \omega_{4}: v \cdot \mathbf{M}=\boldsymbol{\gamma}(s),
\end{aligned}
$$

where $\mathbf{u}_{0}(s)$ and $\boldsymbol{\vartheta}_{0}(s)$ are given functions of the arc length that respectively define the displacements and rotations on a part of the shell contour.

If the strains are small, an example of the constitutive equation is the following quadratic form 


$$
\begin{aligned}
2 W & =\alpha_{1} \operatorname{tr}^{2} \mathbf{e}_{\|}+\alpha_{2} \operatorname{tr} \mathbf{e}_{\|}^{2}+\alpha_{3} \operatorname{tr}\left(\mathbf{e}_{\|} \cdot \mathbf{e}_{\|}^{T}\right)+\alpha_{4} \mathbf{n} \cdot \mathbf{e}^{T} \cdot \mathbf{e} \cdot \mathbf{n} \\
& +\beta_{1} \operatorname{tr}^{2} \kappa_{\|}+\beta_{2} \operatorname{tr} \kappa_{\|}^{2}+\beta_{3} \operatorname{tr}\left(\kappa_{\|} \cdot \kappa_{\|}^{T}\right)+\beta_{4} \mathbf{n} \cdot \kappa^{T} \cdot \kappa \cdot \mathbf{n}
\end{aligned}
$$

This form describes physically linear isotropic shells. Here $\alpha_{k}$ and $\beta_{k}$ are elastic constants $(k=1,2,3,4)$ and

$$
\mathbf{e}_{\|} \triangleq \mathbf{e} \cdot \mathbf{A}, \quad \kappa_{\|} \triangleq \kappa \cdot \mathbf{A} .
$$

Considering Eqs (2.33) and (2.36), the stress tensor and the couple stress tensor are expressed by the formulas

$$
\begin{aligned}
& \mathbf{T}=\alpha_{1} \mathbf{A} \operatorname{tr} \mathbf{e}_{\|}+\alpha_{2} \mathbf{e}_{\|}^{T}+\alpha_{3} \mathbf{e}_{\|}+\alpha_{4} \mathbf{e} \cdot \mathbf{n} \otimes \mathbf{n}, \\
& \mathbf{M}=\beta_{1} \mathbf{A} \operatorname{tr} \boldsymbol{\kappa}_{\|}+\beta_{2} \boldsymbol{\kappa}_{\|}^{T}+\beta_{3} \kappa_{\|}+\beta_{4} \kappa \cdot \mathbf{n} \otimes \mathbf{n} .
\end{aligned}
$$

Supplemented with Eqs (2.34) and (2.35), the linear constitutive equations (2.37), (2.38) constitute the linear boundary-value problem with respect to the fields of displacements and rotations. It describes the equilibrium of the micropolar shell when strains are infinitesimal.

When the strains are small, the Lagrange variational principle (2.26) is transformed to the following form

$$
\mathcal{E}_{1}[\mathbf{u}, \boldsymbol{\vartheta}]=\iint_{\sigma} W(\mathbf{e}, \boldsymbol{\kappa}) \mathrm{d} \sigma-\mathcal{A}[\mathbf{u}, \boldsymbol{\vartheta}],
$$

where the potential of the external loads $\mathcal{A}[\mathbf{u}, \vartheta]$ is defined by the equation

$$
\mathcal{A}[\mathbf{u}, \vartheta] \triangleq \iint_{\sigma}(\mathbf{f} \cdot \mathbf{u}+\mathbf{c} \cdot \vartheta) \mathrm{d} \sigma+\int_{\omega_{2}} \boldsymbol{\varphi} \cdot \mathbf{u} \mathrm{d} s+\int_{\omega_{4}} \gamma \cdot \vartheta \mathrm{d} s .
$$

Let functional (2.39) be given on the set of twice differentiable fields of displacements and rotations of the surface $\sigma$ that satisfy the boundary conditions $(2.35)_{1}$ and $(2.35)_{3}$ on $\omega_{1}$ and $\omega_{3}$, respectively. It is easy to check that the condition of the functional to have a stationary value is equivalent to the equilibrium equations (2.34) and the boundary conditions $(2.35)_{2}$ and $(2.35)_{4}$ on $\omega_{2}$ and $\omega_{4}$, respectively. Let us note that when the strains are small and the form $W(\mathbf{e}, \boldsymbol{\kappa})$ is positive definite, the Lagrange variational principle is a minimal principle, this means functional (2.39) takes a minimal value on the equilibrium solution.

In the linear theory it is valid a variational principle for free oscillations. By linearity, eigen-solutions are proportional to $e^{i \Omega t}\left(\mathbf{u}=\mathbf{u}^{\circ} e^{i \Omega t}, \vartheta=\vartheta^{\circ} e^{i \Omega t}\right)$. Now the variational Rayleigh principle can be formulated: the forms of the eigen-oscillations of the shell are stationary points of the strain energy functional 


$$
\varepsilon_{4}\left[\mathbf{u}^{\circ}, \vartheta^{\circ}\right]=\iint_{\sigma} W\left(\mathbf{e}^{\circ}, \kappa^{\circ}\right) \mathrm{d} \sigma
$$

where

$$
\mathbf{e}^{\circ}=\nabla \mathbf{u}^{\circ}+\mathbf{A} \times \vartheta^{\circ}, \quad \kappa^{\circ}=\nabla \vartheta^{\circ},
$$

on the set of functions that satisfy the following conditions

$$
\omega_{1}: \mathbf{u}^{\circ}=\mathbf{0}, \quad \omega_{3}: \vartheta^{\circ}=\mathbf{0}
$$

and restriction

$$
\iint_{\sigma} \rho K\left(\mathbf{u}^{\circ}, \vartheta^{\circ}\right) \mathrm{d} \sigma=1
$$

Functions $\mathbf{u}^{\circ}, \vartheta^{\circ}$ represent the amplitudes of oscillations for the displacements and small rotations.

The Rayleigh variational principle is equivalent to the stationary principle for the Rayleigh quotient

$$
\mathcal{R}\left[\mathbf{u}^{\circ}, \vartheta^{\circ}\right]=\frac{\iint_{\sigma} W\left(\mathbf{e}^{\circ}, \boldsymbol{\kappa}^{\circ}\right) \mathrm{d} \sigma}{\iint_{\sigma} \rho K\left(\mathbf{u}^{\circ}, \vartheta^{\circ}\right) \mathrm{d} \sigma},
$$

that is defined on kinematically admissible functions $\mathbf{u}^{\circ}, \vartheta^{\circ}$. Note that the least squared eigenfrequency for the shell corresponds the minimal value of $\mathcal{R}$

$$
\Omega_{\min }^{2}=\inf \mathcal{R}\left[\mathbf{u}^{\circ}, \vartheta^{\circ}\right]
$$

on $\mathbf{u}^{\circ}, \vartheta^{\circ}$ satisfying (2.41). Using the Courant minimax principle [14] the Rayleigh quotient (2.43) allows us to estimate the values of higher eigenfrequencies. For this we should consider $\mathcal{R}$ on the set of functions that are orthogonal to the previous modes of eigen-oscillations in some sense.

\subsubsection{Principle Peculiarities of the Micropolar Shell Theory}

Let us summarize principle peculiarities of the shell theory under consideration:

1. The shell equilibrium equation constitute a nonlinear system partial differential equations. In general, the system is elliptic but in some circumstances the ellipticity condition can fail. We will discuss this later.

2. General theorems of existence of equilibrium or dynamic solutions are absent. Moreover, there are examples when under some loads the equilibrium solutions 
does not exist. As for other nonlinear systems, a solution of the equilibrium problem can be non-unique, in general.

3. The Lagrange variational principle is not minimal, it is only a stationary variational principle. The only exception is for the linear theory.

4. For the linear theory of micropolar shells it can be demonstrated the theorems of existence and uniqueness of a solution.

Further developments of this version of the shell theory can be produced in the following directions:

1. Development of mathematical theory that should be based on the methods of partial differential equations theory, functional analysis and calculus of variations.

2. Numerical algorithms for solution of the reduced systems of nonlinear equations. For example, it can be done within the framework of the finite element method, see for example the numerical results in $[10,11,12]$.

3. Analysis of the restrictions of the nonlinear constitutive equations.

4. Extension of the two-dimensional constitutive equations for the shell made of various materials. In particular, the extension can include viscoelasticity, thermal effects, etc. In particular, the theory of thermoelastic and thermoviscoelastic shells with phase transitions is developed in [19].

Some of the above problems will be considered in later sections.

\subsection{Constitutive Restrictions for Micropolar Shells}

In nonlinear elasticity there are well known so-called constitutive restrictions. They are the strong ellipticity condition, the Hadamard inequality, the GCN-condition, and some others $[56,57,58]$. Each of them play some role in nonlinear elasticity. They express mathematically precise and physically intuitive restrictions for constitutive equations of elastic bodies. In particular, the GCN condition proposed by Coleman and Noll asserts "that the transformation from deformation gradient to first Piola-Kirchhoff stress tensor shall be monotone with respect to pairs of deformations differing from one another by a pure stretch" (see, [58]).

The aim of this section is to formulate similar constitutive restrictions in the general nonlinear theory of micropolar shells. Here we formulate the generalized Coleman-Noll inequality (GCN-condition), the strong ellipticity condition of equilibrium equations and the Hadamard inequality. The inequalities represent possible restrictions of constitutive equations of elastic shells under finite deformation. We prove that the Coleman-Noll inequality implies strong ellipticity of shell equilibrium equations. 


\subsubsection{Linear Theory of Micropolar Shells}

In the linear shell theory as well as in the case of the three-dimensional elasticity, it is necessary to establish additional restrictions, so-called constitutive inequalities or constitutive restrictions. Again we stipulate the specific strain energy $W(\mathbf{e}, \boldsymbol{\kappa})$ to be positive definite. Now the energy is a quadratic form of both of the linear strain tensor and the linear bending strain tensor. In particular, for an isotropic shell it takes the form (2.36). Positivity of the quadratic form (2.36) for all values of $\mathbf{e}$ and $\boldsymbol{\kappa}$ is equivalent to the following set of inequalities

$$
\begin{array}{llll}
2 \alpha_{1}+\alpha_{2}+\alpha_{3}>0, & \alpha_{2}+\alpha_{3}>0, & \alpha_{3}-\alpha_{2}>0, & \alpha_{4}>0 \\
2 \beta_{1}+\beta_{2}+\beta_{3}>0, & \beta_{2}+\beta_{3}>0, & \beta_{3}-\beta_{2}>0, & \beta_{4}>0 .
\end{array}
$$

The inequality

$$
W(\mathbf{e}, \boldsymbol{\kappa})>0, \quad \forall \mathbf{e}, \kappa \neq \mathbf{0}
$$

and the following from this the inequalities for the elastic constants of an isotropic material (2.44) are the simplest example of additional inequalities in the shell theory. When they fail it leads to a number of pathological consequences such as nonuniqueness of the solution of boundary value problems of linear shell theory that implies that a solution does not exist for some loads. At second, the propagation of waves in some directions becomes impossible that is not natural from the physical point of view. In the case of finite strains, the positive definiteness of the specific energy $W(\mathbf{E}, \mathbf{K})$ is not a warranty that the desired properties of constitutive equations hold, here must be fulfilled some additional inequalities.

\subsubsection{Coleman-Noll Inequality for Elastic Shells}

Let us suppose that a certain equilibrium state of a nonlinear elastic shell of the Cosserat type under the action of a given load is known. Further we will call it initial or basic stressed state. This state is defined by the vector field $\mathbf{R}\left(q^{\alpha}\right)$ and the tensor field $\mathbf{Q}\left(q^{\alpha}\right)$. Along with the basic stressed state we consider some perturbed equilibrium state which differs from the basic one. The linear parts of increments of different quantities that characterize the perturbed equilibrium we will denote using dots above, for example

$$
\mathbf{D}=\left.\frac{\mathrm{d}}{\mathrm{d} \tau} \mathbf{D}[\nabla(\mathbf{R}+\tau \mathbf{u}, \mathbf{Q}-\tau \mathbf{Q} \times \boldsymbol{\theta}, \nabla(\mathbf{Q}-\tau \mathbf{Q} \times \boldsymbol{\theta}))]\right|_{\tau=0} .
$$

Here $\mathbf{u}$ is the vector of the additional infinitesimal displacement, while $\theta$ is the vector of the additional infinitesimal rotation characterizing the small rotation with respect to the initial stressed state. The following relations are valid

$$
\mathbf{R}=\mathbf{u}, \quad \mathbf{Q}=-\mathbf{Q} \times \boldsymbol{\theta}, \quad \mathbf{E}=\mathbf{F} \cdot \boldsymbol{\varepsilon} \cdot \mathbf{Q}^{T}, \quad \mathbf{K}=\mathbf{F} \cdot \varkappa \cdot \mathbf{Q}^{T},
$$




$$
\boldsymbol{\varepsilon}=\nabla \mathbf{u}+\mathbf{A} \times \boldsymbol{\theta}, \quad \varkappa=\nabla \theta
$$

where $\varepsilon$ is the strain tensor and $\varkappa$ is the bending strain tensor usually used in the linear shell theory of the Cosserat-type [23, 36, 60].

Note that as the reference configuration may be chosen any stressed state of the shell. To avoid awkward expressions and to simplify the calculations let us assume that the reference configuration coincides with the initial (basic) stressed state of the shell. This means that in the reference configuration $\mathbf{F}=\mathbf{E}=\mathbf{I}-\mathbf{n} \otimes \mathbf{n}, \mathbf{Q}=\mathbf{I}$, $\mathbf{K}=\mathbf{0}$. Under this choice of the reference configuration and using Eqs (2.9), (2.13), (2.45)-(2.47) we obtain

$$
\begin{aligned}
& \mathbf{D}=\frac{\partial^{2} W}{\partial \mathbf{E} \partial \mathbf{E}} \bullet \varepsilon+\frac{\partial^{2} W}{\partial \mathbf{E} \partial \mathbf{K}} \bullet \varkappa-\mathbf{T} \times \theta, \\
& \mathbf{G}=\frac{\partial^{2} W}{\partial \mathbf{K} \partial \mathbf{E}} \bullet \varepsilon+\frac{\partial^{2} W}{\partial \mathbf{K} \partial \mathbf{K}} \bullet \varkappa-\mathbf{M} \times \theta .
\end{aligned}
$$

Here and below we use the operation of a scalar product of tensors given by the formulae

$$
\begin{gathered}
\left(C^{k s t p} \mathbf{R}_{k} \otimes \mathbf{R}_{s} \otimes \mathbf{R}_{t} \otimes \mathbf{R}_{p}\right) \bullet\left(U_{m n} \mathbf{R}^{m} \otimes \mathbf{R}^{n}\right) \triangleq C^{k s m n} U_{m n} \mathbf{R}_{k} \otimes \mathbf{R}_{s}, \\
\left(V_{i j} \mathbf{R}^{i} \otimes \mathbf{R}^{j}\right) \bullet\left(C^{k s t p} \mathbf{R}_{k} \otimes \mathbf{R}_{s} \otimes \mathbf{R}_{t} \otimes \mathbf{R}_{p}\right) \bullet\left(U_{m n} \mathbf{R}^{m} \otimes \mathbf{R}^{n}\right) \triangleq C^{i j m n} V_{i j} U_{m n} .
\end{gathered}
$$

Suppose that the external couples in the initial and perturbed stressed states of the shell vanish $\mathbf{c}=\boldsymbol{\gamma}=\mathbf{0}$, while the external forces are "dead". Then the elastic energy of the shell is given by the relation

$$
\Pi \equiv \mathcal{E}_{1}=\iint_{\sigma} W \mathrm{~d} \sigma-\iint_{\sigma} \mathbf{f} \cdot(\mathbf{R}-\mathbf{r}) \mathrm{d} \sigma-\int_{\omega_{2}} \varphi \cdot(\mathbf{R}-\mathbf{r}) \mathrm{d} s .
$$

Let us consider the energy increment in the perturbed equilibrium state with respect to the energy in the initial state taking into account terms of order one and two

$$
\Pi-\Pi_{0}=\tau\left(\frac{\mathrm{d} \Pi}{\mathrm{d} \tau}\right)_{\tau=0}+\frac{1}{2} \tau^{2}\left(\frac{\mathrm{d}^{2} \Pi}{\mathrm{d} \tau^{2}}\right)_{\tau=0}+\ldots
$$

According to the constitutive relations of an elastic shell (2.9) and Eqs (2.46), (2.47) we obtain

$$
\begin{gathered}
\frac{\mathrm{d} \Pi}{\mathrm{d} \tau}=\iint_{\sigma}\left[\operatorname{tr}\left(\mathbf{D}^{T} \cdot \nabla \mathbf{u}\right)+\operatorname{tr}\left(\mathbf{D}^{T} \cdot \mathbf{F} \times \boldsymbol{\theta}\right)+\operatorname{tr}\left(\mathbf{G}^{T} \cdot \nabla \boldsymbol{\theta}\right)\right] \mathrm{d} \sigma \\
-\iint_{\sigma} \mathbf{f} \cdot \mathbf{u} \mathrm{d} \sigma-\int_{\omega_{2}} \boldsymbol{\varphi} \cdot \mathbf{u} \mathrm{d} s .
\end{gathered}
$$


Differentiating Eq. (2.49) with respect to the parameter $\tau$ and taking into account that the reference configuration coincides with the basic stressed state and Eqs (2.46) we get

$$
\begin{gathered}
\left.\frac{\mathrm{d}^{2} \Pi}{\mathrm{d} \tau^{2}}\right|_{\tau=0}=\iint_{\Sigma}\left[\operatorname{tr}\left(\mathbf{D}^{T} \cdot \nabla \mathbf{u}\right)+\operatorname{tr}\left(\mathbf{D}^{T} \times \theta\right)\right. \\
\left.+\operatorname{tr}\left(\mathbf{T}^{T} \cdot(\nabla \mathbf{u}) \times \boldsymbol{\theta}\right)+\operatorname{tr}\left(\mathbf{G}^{\cdot T} \cdot \varkappa\right)\right] \mathrm{d} \Sigma .
\end{gathered}
$$

From the equilibrium condition of the basic state and considering Eqs (2.8) and (2.10) it follows that the first variation of the energy

$$
\left.\frac{\mathrm{d} \Pi}{\mathrm{d} \tau}\right|_{\tau=0}
$$

vanishes, and the second variation due to Eqs (2.47) and (2.48) could be represented as

$$
\begin{gathered}
\left.\frac{\mathrm{d}^{2} \Pi}{\mathrm{d} \tau^{2}}\right|_{\tau=0}=2 \iint_{\Sigma} w \mathrm{~d} \Sigma, \quad w=w^{\prime}+w^{\prime \prime}, \\
w^{\prime}=\frac{1}{2} \varepsilon \bullet \frac{\partial^{2} W}{\partial \mathbf{E} \partial \mathbf{E}} \bullet \varepsilon+\varepsilon \bullet \frac{\partial^{2} W}{\partial \mathbf{E} \partial \mathbf{K}} \bullet \varkappa+\frac{1}{2} \varkappa \bullet \frac{\partial^{2} W}{\partial \mathbf{K} \partial \mathbf{K}} \bullet \varkappa, \\
w^{\prime \prime}=\operatorname{tr}\left(\theta \times \mathbf{T}^{T} \cdot \varepsilon\right)-\frac{1}{2} \operatorname{tr}\left(\boldsymbol{\theta} \times \mathbf{T}^{T} \times \theta\right)+\frac{1}{2} \operatorname{tr}\left(\boldsymbol{\theta} \times \mathbf{M}^{T} \cdot \varkappa\right) .
\end{gathered}
$$

The quantity $w$ describes the increment of the elastic energy of the initially prestressed shell under additional infinitesimal deformations. According to Eqs (2.50) and (2.51) this energy may be decomposed into two parts: the energy of pure strains $w^{\prime}$ and the energy of rotations $w^{\prime \prime}$. The coefficients in the quadratic form $w^{\prime \prime}$ are expressed in terms of the stress and couple stress tensors of the initial stressed state and do not depend on material properties of the shell. If the basic stressed state of the shell is natural $(\mathbf{T}=\mathbf{M}=\mathbf{0})$, then $w=w^{\prime}$ and the energy density reduces to the quadratic form of tensors $\varepsilon$ and $\varkappa$. If the decomposition (2.50) and the formulae (2.51) are compared with the similar results on increment of 3D strain energy density [21] of micropolar body one can easily note that they coincide up to notation.

One of the well-known in the nonlinear elasticity $[56,57,58]$ constitutive inequalities is the Coleman-Noll inequality. The differential form of the Coleman-Noll inequality (so-called GCN-condition) expresses the property that the elastic energy density under arbitrary infinitesimal non-zero pure strains and bending strains for any arbitrary reference configuration should be positive. It is necessary to mention that this inequality does not restrict the constitutive equations with regard to any rotations.

Taking into account the energy decomposition (2.50) we obtain an analogue of the Coleman-Noll inequality for elastic shells of the Cosserat type

$$
w^{\prime}(\boldsymbol{\varepsilon}, \varkappa)>0 \quad \forall \boldsymbol{\varepsilon} \neq \mathbf{0}, \quad \boldsymbol{\varkappa} \neq \mathbf{0} .
$$


Using Eqs (2.51) inequality (2.52) can be written in the equivalent form

$$
\left.\frac{\mathrm{d}^{2}}{\mathrm{~d} \tau^{2}} W(\mathbf{E}+\tau \varepsilon, \mathbf{K}+\tau \varkappa)\right|_{\tau=0}>0 \quad \forall \varepsilon \neq \mathbf{0}, \quad \varkappa \neq \mathbf{0} .
$$

Condition (2.53) satisfies the principle of material frame-indifference and could serve as a constitutive inequality for elastic shells.

\subsubsection{Strong Ellipticity and Hadamard Inequality}

The famous constitutive inequalities in the nonlinear elasticity are the strong ellipticity condition and its weak form known as the Hadamard inequality. Following the theory of systems of partial differential equations (PDE) [27, 40] in this section we formulate the strong ellipticity condition of the equilibrium equations (2.8). In the case of dead loading the linearized equilibrium equations have the form

$$
\nabla \cdot \mathbf{D}=\mathbf{0}, \quad \nabla \cdot \mathbf{G}+\left[\mathbf{F}^{T} \cdot \mathbf{D}+(\nabla \mathbf{u})^{T} \cdot \mathbf{D}\right]_{\times}=\mathbf{0},
$$

where $\mathbf{D}^{*}$ and $\mathbf{G}$ are given by formulae similar to Eqs (2.45). Equations (2.54) are the system of linear PDE of second order with respect to $\mathbf{u}$ and $\boldsymbol{\theta}$. If we keep in Eqs (2.54) the differential operators of second order we obtain the relations

$$
\begin{aligned}
& \nabla \cdot\left\{\left[\frac{\partial^{2} W}{\partial \mathbf{E} \partial \mathbf{E}} \bullet\left((\nabla \mathbf{u}) \cdot \mathbf{Q}^{T}\right)+\frac{\partial^{2} W}{\partial \mathbf{E} \partial \mathbf{K}} \bullet\left((\nabla \boldsymbol{\theta}) \cdot \mathbf{Q}^{T}\right)\right] \cdot \mathbf{Q}\right\}, \\
& \boldsymbol{\nabla} \cdot\left\{\left[\frac{\partial^{2} W}{\partial \mathbf{K} \partial \mathbf{E}} \bullet\left((\nabla \mathbf{u}) \cdot \mathbf{Q}^{T}\right)+\frac{\partial^{2} W}{\partial \mathbf{K} \partial \mathbf{K}} \bullet\left((\nabla \boldsymbol{\theta}) \cdot \mathbf{Q}^{T}\right)\right] \cdot \mathbf{Q}\right\},
\end{aligned}
$$

which allow to construct the condition of strong ellipticity of the system (2.54). Using the formal procedure $[27,40]$ we replace the differential operator $\nabla$ by the unit vector $\boldsymbol{v}$ tangential to the surface $\sigma$, while the vector fields $\mathbf{u}$ and $\theta$ by the vectors $\mathbf{a}$ and $\mathbf{b}$, respectively. Thus, we have the algebraic expressions

$$
\begin{aligned}
& v \cdot\left\{\left[\frac{\partial^{2} W}{\partial \mathbf{E} \partial \mathbf{E}} \bullet\left(\boldsymbol{v} \otimes \mathbf{a} \cdot \mathbf{Q}^{T}\right)+\frac{\partial^{2} W}{\partial \mathbf{E} \partial \mathbf{K}} \bullet\left(\boldsymbol{v} \otimes \mathbf{b} \cdot \mathbf{Q}^{T}\right)\right] \cdot \mathbf{Q}\right\}, \\
& v \cdot\left\{\left[\frac{\partial^{2} W}{\partial \mathbf{K} \partial \mathbf{E}} \bullet\left(\boldsymbol{v} \otimes \mathbf{a} \cdot \mathbf{Q}^{T}\right)+\frac{\partial^{2} W}{\partial \mathbf{K} \partial \mathbf{K}} \bullet\left(\boldsymbol{v} \otimes \mathbf{b} \cdot \mathbf{Q}^{T}\right)\right] \cdot \mathbf{H}\right\} .
\end{aligned}
$$

Let us multiply the first equation by the vector a while the second one by the vector $\mathbf{b}$ and add these expressions. Then we obtain the strong ellipticity condition of Eqs (2.54) in the following form

$$
\boldsymbol{v} \cdot\left\{\left[\frac{\partial^{2} W}{\partial \mathbf{E} \partial \mathbf{E}} \bullet\left(\boldsymbol{v} \otimes \mathbf{a} \cdot \mathbf{Q}^{T}\right)+\frac{\partial^{2} W}{\partial \mathbf{E} \partial \mathbf{K}} \bullet\left(\boldsymbol{v} \otimes \mathbf{b} \cdot \mathbf{Q}^{T}\right)\right] \cdot \mathbf{Q}\right\} \cdot \mathbf{a}
$$




$$
+\boldsymbol{v} \cdot\left\{\left[\frac{\partial^{2} W}{\partial \mathbf{E} \partial \mathbf{E}} \bullet\left(\boldsymbol{v} \otimes \mathbf{a} \cdot \mathbf{Q}^{T}\right)+\frac{\partial^{2} W}{\partial \mathbf{K} \partial \mathbf{K}} \bullet\left(\boldsymbol{v} \otimes \mathbf{b} \cdot \mathbf{Q}^{T}\right)\right] \cdot \mathbf{Q}\right\} \cdot \mathbf{b}>0,
$$

$$
\forall \mathbf{a}, \mathbf{b} \neq \mathbf{0} .
$$

Using operation $\bullet$ we can transform the latter equations as follows

$$
\begin{gathered}
\left(\boldsymbol{v} \otimes \mathbf{a} \cdot \mathbf{Q}^{T}\right) \cdot \frac{\partial^{2} W}{\partial \mathbf{E} \partial \mathbf{E}} \bullet\left(\boldsymbol{v} \otimes \mathbf{a} \cdot \mathbf{H}^{T}\right)+2\left(\boldsymbol{v} \otimes \mathbf{a} \cdot \mathbf{Q}^{T}\right) \cdot \frac{\partial^{2} W}{\partial \mathbf{E} \partial \mathbf{K}} \bullet\left(\boldsymbol{v} \otimes \mathbf{b} \cdot \mathbf{Q}^{T}\right) \\
+\left(\boldsymbol{v} \otimes \mathbf{b} \cdot \mathbf{H}^{T}\right) \bullet \frac{\partial^{2} W}{\partial \mathbf{K} \partial \mathbf{E}} \bullet\left(\boldsymbol{v} \otimes \mathbf{b} \cdot \mathbf{Q}^{T}\right)>0, \quad \forall \mathbf{a}, \mathbf{b} \neq \mathbf{0} .
\end{gathered}
$$

This inequality can be written in a more compact form using the matrix notation

$$
\xi \cdot \mathbb{A}(\boldsymbol{v}) \cdot \boldsymbol{\xi}>0, \quad \forall \boldsymbol{v} \in \mathscr{T}_{x} \sigma, \quad v \neq \mathbf{0}, \quad \forall \boldsymbol{\xi} \in \mathbb{R}^{6}, \quad \boldsymbol{\xi} \neq \mathbf{0},
$$

where $\xi=\left(\mathbf{a}^{\prime}, \mathbf{b}^{\prime}\right) \in \mathbb{R}^{6}, \mathbf{a}^{\prime}=\mathbf{a} \cdot \mathbf{Q}^{T}, \mathbf{b}^{\prime}=\mathbf{b} \cdot \mathbf{Q}^{T}$, and the matrix $\mathbb{A}(v)$ is given by

$$
\mathbf{A}(\boldsymbol{v}) \triangleq\left[\begin{array}{cc}
\frac{\partial^{2} W}{\partial \mathbf{E} \partial \mathbf{E}}\{\boldsymbol{v}\} & \frac{\partial^{2} W}{\partial \mathbf{E} \partial \mathbf{K}}\{v\} \\
\frac{\partial^{2} W}{\partial \mathbf{K} \partial \mathbf{E}}\{v\} & \frac{\partial^{2} W}{\partial \mathbf{K} \partial \mathbf{K}}\{v\}
\end{array}\right],
$$

where for any fourth-order tensor $\mathbf{K}$ and any vector $v: \mathbf{K}\{v\} \triangleq K_{k l m n} v_{k} v_{m} \mathbf{i}_{l} \otimes \mathbf{i}_{n}$. The inequality (2.55) is the strong ellipticity condition of the equilibrium equations (2.8) of an elastic shells. A weak form of the inequality (2.55) is an analogue of the Hadamard inequality for the shell. These inequalities are examples of possible restrictions of the constitutive equations of elastic shells under finite deformations. As in the case of simple materials, a break in inequality the (2.55) means the possibility of existing non-smooth solutions of the equilibrium equations (2.8).

The strong ellipticity condition may be written in the equivalent form

$$
\left.\frac{\mathrm{d}^{2}}{\mathrm{~d} \tau^{2}} W\left(\mathbf{E}+\tau \boldsymbol{v} \otimes \mathbf{a}^{\prime}, \mathbf{K}+\tau v \otimes \mathbf{b}^{\prime}\right)\right|_{\tau=0}>0 \quad \forall v, \mathbf{a}^{\prime}, \mathbf{b}^{\prime} \neq \mathbf{0} .
$$

Comparing the condition of strong ellipticity (2.56) and the Coleman-Noll inequality (2.53) one can see that the latter implies the former. Indeed, the inequality (2.53) holds for any tensors $\varepsilon$ and $\varkappa$. Note that the tensors $\varepsilon$ and $\varkappa$ may by nonsymmetric, in general. If we substitute in the inequality (2.53) the relations $\varepsilon=\boldsymbol{v} \otimes \mathbf{a}^{\prime}$ and $\varkappa=\boldsymbol{v} \otimes \mathbf{b}^{\prime}$ then we immediately obtain the inequality (2.56). Thus, the strong ellipticity condition is the special case of the Coleman-Noll inequality. This is the essential distinction between the shell theory of the Cosserat type and the theory of simple elastic materials $[57,58]$ where these two properties completely independent of each other in the sense that neither of them implies the other one. For 3D elastic micropolar media the strong ellipticity and the Hadamard conditions are formulated in [21]. 
In the shell theory it is widely used the following particular constitutive equation

$$
W(\mathbf{E}, \mathbf{K})=W_{1}(\mathbf{E})+W_{2}(\mathbf{K}) .
$$

For example, the constitutive relations (2.18) have the form (2.57).

Now condition (2.55) is equivalent to two more simple inequalities

$$
\begin{gathered}
\mathbf{a} \cdot \frac{\partial^{2} W_{1}}{\partial \mathbf{E} \partial \mathbf{E}}\{\boldsymbol{v}\} \cdot \mathbf{a}>0, \quad \mathbf{b} \cdot \frac{\partial^{2} W_{2}}{\partial \mathbf{K} \partial \mathbf{K}}\{v\} \cdot \mathbf{b}>0, \\
\forall v, \quad v \neq \mathbf{0}, \quad v \cdot \mathbf{n}=0, \quad \forall \mathbf{a}, \mathbf{b} \in \mathbb{R}^{3}, \quad \mathbf{a}, \mathbf{b} \neq \mathbf{0} .
\end{gathered}
$$

As an example, let us consider when for constitutive equation (2.18) the conditions (2.55) are valid. It can be shown that the second-order tensors

$$
\frac{\partial^{2} W_{1}}{\partial \mathbf{E} \partial \mathbf{E}}\{\boldsymbol{v}\} \text { and } \frac{\partial^{2} W_{2}}{\partial \mathbf{K} \partial \mathbf{K}}\{\boldsymbol{v}\}
$$

are given by formulas

$$
\begin{aligned}
& \frac{\partial^{2} W_{1}}{\partial \mathbf{E} \partial \mathbf{E}}\{v\}=\alpha_{3} \mathbf{A}+\left(\alpha_{1}+\alpha_{2}\right) \mathbf{v} \otimes v+\alpha_{4} \mathbf{n} \otimes \mathbf{n}, \\
& \frac{\partial^{2} W_{2}}{\partial \mathbf{K} \partial \mathbf{K}}\{v\}=\beta_{3} \mathbf{A}+\left(\beta_{1}+\beta_{2}\right) v \otimes v+\beta_{4} \mathbf{n} \otimes \mathbf{n} .
\end{aligned}
$$

Inequality (2.55) is valid under the following conditions

$$
\begin{array}{ccc}
\alpha_{3}>0, & \alpha_{1}+\alpha_{2}+\alpha_{3}>0, & \alpha_{4}>0, \\
\beta_{3}>0, & \beta_{1}+\beta_{2}+\beta_{3}>0, & \beta_{4}>0 .
\end{array}
$$

For an linear isotropic shell, the inequalities (2.59) provide strong ellipticity of equilibrium equations (2.34), they are more weak in comparison with the condition of positive definiteness of (2.44). If we consider the constitutive equations of an isotropic micropolar shell (2.18) then the inequality (2.55) reduces to the system of inequalities (2.59).

\subsubsection{Strong Ellipticity Condition and Acceleration Waves}

Using approach $[3,15,22]$, we show that the inequality $(2.55)$ coincides with the conditions for propagation of acceleration waves in a shell. We consider a motion that may be accompanied by a jump in the continuity of kinematic and dynamic quantities on a certain smooth curve $C(t)$, which is called singular (Fig. 2.2). We assume that the limit values of these quantities exist on $C$ and that they are generally 
different on the opposite sides of $C$. The jump of an arbitrary quantity $\boldsymbol{\Psi}$ on $C$ is denoted by $[[\boldsymbol{\Psi}]]=\boldsymbol{\Psi}^{+}-\boldsymbol{\Psi}^{-}$.

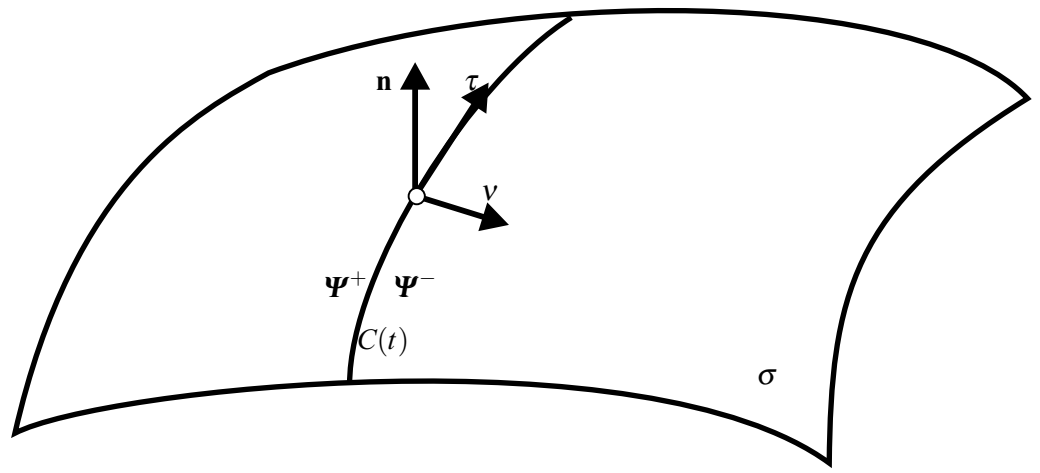

Fig. 2.2 Singular curve

The acceleration wave (weak-discontinuity wave or second-order singular curve) is a moving singular curve $C$ on which the second derivatives (with respect to the spatial coordinates and time) of the radius-vector $\mathbf{R}$ and the microrotation tensor $\mathbf{Q}$ are discontinuous, while the quantities themselves and their first derivatives are continuous, this means

$$
[[\mathbf{F}]]=\mathbf{0}, \quad[[\nabla \mathbf{Q}]=\mathbf{0}, \quad \llbracket[\mathbf{v}]]=\mathbf{0}, \quad[[\boldsymbol{\omega} \rrbracket=\mathbf{0}
$$

are valid on $C$. According to Eqs (2.2), the strain measure $\mathbf{E}$ and the bending strain tensor $\mathbf{K}$ are continuous near $C$, and, with respect to constitutive equations (2.9), jumps of the tensors $\mathbf{D}$ and $\mathbf{G}$ are absent. The application of the Maxwell theorem [57] to continuous fields of velocities $\mathbf{v}$ and $\omega$, surface stress tensor $\mathbf{D}$, and the surface couple stress tensor $\mathbf{G}$ yields a system of equations that relate the jumps of their derivatives with respect to the spatial coordinates and time

$$
\begin{gathered}
\llbracket \frac{d \mathbf{v}}{d t} \rrbracket=-V \mathbf{a}, \quad\left[[\nabla \mathbf{v}]=v \otimes \mathbf{a}, \quad \llbracket \frac{d \boldsymbol{\omega}}{d t} \rrbracket=-V \mathbf{b}, \quad \llbracket \nabla \omega\right]=\mathbf{v} \otimes \mathbf{b}, \\
V[\nabla \nabla \cdot \mathbf{D}]]=-\boldsymbol{v} \cdot \llbracket \frac{d \mathbf{D}}{d t} \rrbracket, \quad V\left[\left[\nabla \cdot \mathbf{G} \rrbracket=-\boldsymbol{v} \cdot \llbracket \frac{d \mathbf{G}}{d t} \rrbracket .\right.\right.
\end{gathered}
$$

Here $\mathbf{a}$ and $\mathbf{b}$ are the vector amplitudes for the jumps of the linear and angular accelerations, $\boldsymbol{v}$ is the unit normal vector to $C$ such that $\mathbf{n} \cdot \boldsymbol{v}=0$, and $V$ is the velocity of the surface $C$ in the direction $\boldsymbol{v}$. If external forces and couples are continuous, the relations

$$
[[\nabla \cdot \mathbf{D}]]=\rho \llbracket d \frac{\mathbf{K}_{1}}{d t} \rrbracket, \quad[[\boldsymbol{\nabla} \cdot \mathbf{G}]]=\rho \gamma \llbracket \frac{d \mathbf{K}_{2}}{d t} \rrbracket
$$

follow immediately from the equations of motion (2.14). 
Differentiating constitutive Eqs (2.9) and using equations (2.60) and (2.61), we express latter relations only in terms of the vector amplitudes $\mathbf{a}$ and $\mathbf{b}$

$$
\begin{array}{r}
\boldsymbol{v} \cdot \frac{\partial^{2} W}{\partial \mathbf{E} \partial \mathbf{Y}} \bullet\left(v \otimes \mathbf{a} \cdot \mathbf{Q}^{T}\right)+v \cdot \frac{\partial^{2} W}{\partial \mathbf{E} \partial \mathbf{L}} \bullet\left(v \otimes \mathbf{b} \cdot \mathbf{Q}^{T}\right)=\rho V^{2}\left[\mathbf{a} \cdot \mathbf{Q}^{T}\right. \\
\left.+\left(\mathbf{Q} \cdot \Theta_{1}^{T} \cdot \mathbf{Q}^{T}\right) \cdot\left(\mathbf{b} \cdot \mathbf{Q}^{T}\right)\right], \\
\boldsymbol{v} \cdot \frac{\partial^{2} W}{\partial \mathbf{K} \partial \mathbf{E}} \bullet\left(\boldsymbol{v} \otimes \mathbf{a} \cdot \mathbf{Q}^{T}\right)+v \cdot \frac{\partial^{2} W}{\partial \mathbf{K} \partial \mathbf{L}} \bullet\left(\boldsymbol{v} \otimes \mathbf{b} \cdot \mathbf{Q}^{T}\right)= \\
\rho V^{2}\left[\left(\mathbf{Q} \cdot \boldsymbol{\Theta}_{1} \cdot \mathbf{Q}^{T}\right) \cdot\left(\mathbf{a} \cdot \mathbf{Q}^{T}\right)\right. \\
\left.+\left(\mathbf{Q} \cdot \boldsymbol{\Theta}_{2} \cdot \mathbf{Q}^{T}\right) \cdot\left(\mathbf{b} \cdot \mathbf{Q}^{T}\right)\right] .
\end{array}
$$

Hence the strong ellipticity condition is valid these relations can be also written in a more compact form

$$
\mathbb{A}(\boldsymbol{v}) \cdot \boldsymbol{\xi}=\rho V^{2} \mathbb{B} \cdot \boldsymbol{\xi}
$$

where the matrix $\mathbb{B}$ is given by

$$
\mathbf{B}=\left[\begin{array}{cc}
\mathbf{I} & \mathbf{Q} \cdot \Theta_{1}^{T} \cdot \mathbf{Q}^{T} \\
\mathbf{Q} \cdot \Theta_{1} \cdot \mathbf{Q}^{T} & \mathbf{Q} \cdot \Theta_{2} \cdot \mathbf{Q}^{T}
\end{array}\right]
$$

Thus, the problem of acceleration wave propagation in the shell has been reduced to the spectral problem given by the algebraic equations (2.62). Owing to the existence of the potential-energy function $W, \mathbb{A}(\boldsymbol{v})$ is symmetric. Matrix $\mathbb{B}$ is also symmetric and positive definite. This property enables to formulate an analogue of the Fresnel-Hadamard-Duhem theorem for the elastic shell:

Theorem 2.1. The squares of the velocities of a second order singular curve (acceleration wave) in the elastic shell are real for arbitrary propagation directions specified by the vector $v$.

Note that the positive definiteness of $\mathbb{A}(\boldsymbol{v})$, which is necessary and sufficient for the wave velocity $V$ to be real, coincides with the strong ellipticity inequality (2.55).

For a physically linear shell, let us present an example of solution of problem (2.62). Suppose that $\Theta_{1}$ is zero and $\Theta_{2}$ is the spherical part of the tensor, that is $\boldsymbol{\Theta}_{2}=\imath \mathbf{l}$ ), where $\imath$ is the rotatory inertia measure. Let us assume that the inequalities (2.59) are valid. Then solutions of equation (2.62) are 


$$
\begin{gathered}
U_{1}=\sqrt{\frac{\alpha_{3}}{\rho}}, \quad \xi_{1}=(\tau, \mathbf{0}), \quad U_{2}=\sqrt{\frac{\alpha_{1}+\alpha_{2}+\alpha_{3}}{\rho}}, \quad \xi_{2}=(\boldsymbol{v}, \mathbf{0}), \\
U_{3}=\sqrt{\frac{\alpha_{4}}{\rho}}, \quad \xi_{3}=(\mathbf{n}, \mathbf{0}), \quad U_{4}=\sqrt{\frac{\beta_{3}}{\rho \imath}}, \quad \xi_{4}=(\mathbf{0}, \boldsymbol{\tau}), \\
U_{5}=\sqrt{\frac{\beta_{1}+\beta_{2}+\beta_{3}}{\rho \imath}}, \quad \xi_{5}=(\mathbf{0}, \boldsymbol{v}), \quad U_{6}=\sqrt{\frac{\beta_{4}}{\rho \imath}}, \quad \xi_{6}=(\mathbf{0}, \mathbf{n}) .
\end{gathered}
$$

Solutions (2.63) describe transversal and longitudinal waves of acceleration and microrotation accelerations.

\subsubsection{Ordinary Ellipticity}

When equilibrium equations are not elliptic it may result in the break of continuity of solutions. Let us consider this in more detail. We will assume singular timeindependent curves of the second order. Suppose on the shell surface $\sigma$ there exists a curve $\gamma$ on which a jump in the second derivatives of the position vector $\mathbf{R}$ or microrotation tensor $\mathbf{Q}$ happens. We will call such a jump the weak discontinuity. For example, the curvature of $\Sigma$ is determined through second derivatives of $\mathbf{R}$ so such discontinuity can exhibited in the form of sharp bends of the shell surface.

From the equilibrium equations it follows

$$
[[\nabla \cdot \mathbf{D}]]=\mathbf{0}, \quad[\nabla \cdot \mathbf{G}]]=\mathbf{0} .
$$

Repeating transformations of the previous section, we can reduce these to

$$
\left[\begin{array}{c}
\frac{\partial^{2} W}{\partial \mathbf{E} \partial \mathbf{E}}\{\boldsymbol{v}\} \frac{\partial^{2} W}{\partial \mathbf{E} \partial \mathbf{K}}\{\boldsymbol{v}\} \\
\frac{\partial^{2} W}{\partial \mathbf{K} \partial \mathbf{E}}\{\boldsymbol{v}\} \frac{\partial^{2} W}{\partial \mathbf{K} \partial \mathbf{L}}\{\boldsymbol{v}\}
\end{array}\right] \cdot\left[\begin{array}{l}
\mathbf{a}^{\prime} \\
\mathbf{b}^{\prime}
\end{array}\right]=\mathbf{0}
$$

We can rewrite this in a more compact form

$$
\mathbb{A}(v) \cdot \xi=\mathbf{0}, \quad \xi=\left(\mathbf{a}^{\prime}, \mathbf{b}^{\prime}\right) \in \mathbb{R}^{6} .
$$

The existence of non-trivial solutions of Eq. (2.64) means that weak discontinuities arise. The condition for this is the determinant of matrix $\mathbb{A}(\boldsymbol{v})$ should be zero. When there holds

$$
\operatorname{det} \mathbf{A}(v) \neq 0,
$$

such discontinuities are impossible.

For the constitutive equation of the form

$$
W=W_{1}(\mathbf{E})+W_{2}(\mathbf{K})
$$


condition (2.65) splits into two conditions

$$
\operatorname{det} \frac{\partial^{2} W_{1}}{\partial \mathbf{E} \partial \mathbf{E}}\{\boldsymbol{v}\} \neq 0, \quad \operatorname{det} \frac{\partial^{2} W_{1}}{\partial \mathbf{K} \partial \mathbf{K}}\{\boldsymbol{v}\} \neq 0 .
$$

As an example, we consider the conditions (2.65) for the constitutive equations of a physically linear shell (2.18). With the use of Eqs (2.58) we can show the conditions (2.66) reduce to the inequalities

$$
\alpha_{3} \neq 0, \quad \alpha_{1}+\alpha_{2}+\alpha_{3} \neq 0, \quad \alpha_{4} \neq 0, \quad \beta_{3} \neq 0, \quad \beta_{1}+\beta_{2}+\beta_{3} \neq 0, \quad \beta_{4} \neq 0 .
$$

Condition (2.65) is the ellipticity condition of the equilibrium equations of shell theory (ellipticity in sense of Petrovsky). The condition follows from the general definition of ellipticity in the theory of partial differential equations. Condition (2.65) is also called the ordinary ellipticity condition, which is more weak than the strong ellipticity condition (2.55).

\subsection{Phase Equilibrium Conditions in Micropolar Shells}

In the shell material can arise structural or phase transitions when it is produced or being in exploitation. For example, such transformations can happen in thin-walled structures made of polymer material or memory shape alloys. Various phase transitions can happen in biology membranes or liquid crystal films. In this chapter, we restrict our consideration by the frontal phase transitions, that is the phase transitions with a sharp border between the phases. We consider these transitions that are due to the strain change under constant temperature. Let deformation process to be isothermal or adiabatic. A practically important example of use of thin-walled structures gives us the use of the mono- or polycrystal films of the alloys of the type $\mathrm{NiTi}$, that is $\mathrm{NiMnGa}, \mathrm{NiTiCu}, \mathrm{NiAl}$, etc., which posses the shape memory effect, see e.g. [7, 46]. Such films are used in microelectromechanical systems, MEMS, such as miniature pumps, microengines, etc. One of the first mechanical models of deformations of martensitic films is proposed in [8,34], see also [7, 46], where, in particular, as a result of a special reduction procedure from a three-dimensional layer to a surface, a model of a directed membrane is elaborated, that is of the shell that does not possesses bending rigidity. Its constitutive equation depends on the displacement field and its derivatives but besides, on the director field that is kinematically independent of the displacement field. Within the nonlinear shell theory, investigation of equilibrium of phases is presented in [17] and extended in [54] to the influence of line tension effects. The quasistatic deformation of two-phase thermoelastic and thermoviscoelastic shells are considered in $[19,20]$. Within the framework of the theory of biological membranes the phase transitions in 2D structures are investigated in $[2,9]$. 


\subsubsection{Thermodynamic Continuity Conditions}

For the sake of simplicity we restrict ourselves by the case of thermodynamic equilibrium. This means that we can use the Gibbs' variational approach [28]. Let us suppose the phase transition happens along the whole shell thickness simultaneously. This allows us to introduce a curve on the shell surface that separates the material phases with different properties. The assumption is based on experimental data for deformation of thin plates and thin-walled tubes with shape memory effect, cf. [31, 32, 33, 37].

For a two-phase shell, surface $\sigma$ consists of two parts, $\sigma_{-}$and $\sigma_{+}$(cf. Fig. 2.3), that are separated by a smooth curve $\gamma$. Curve $\gamma$ is unknown in advance, it is a preimage of the phase transition border. In the actual configuration $\gamma$ is represented by $\Gamma$. We should find the shell deformation (2.1) together with $\gamma$. Let us note $\gamma$ is a particular case of the singular curve on which the continuity conditions for some quantities under consideration can be violated.

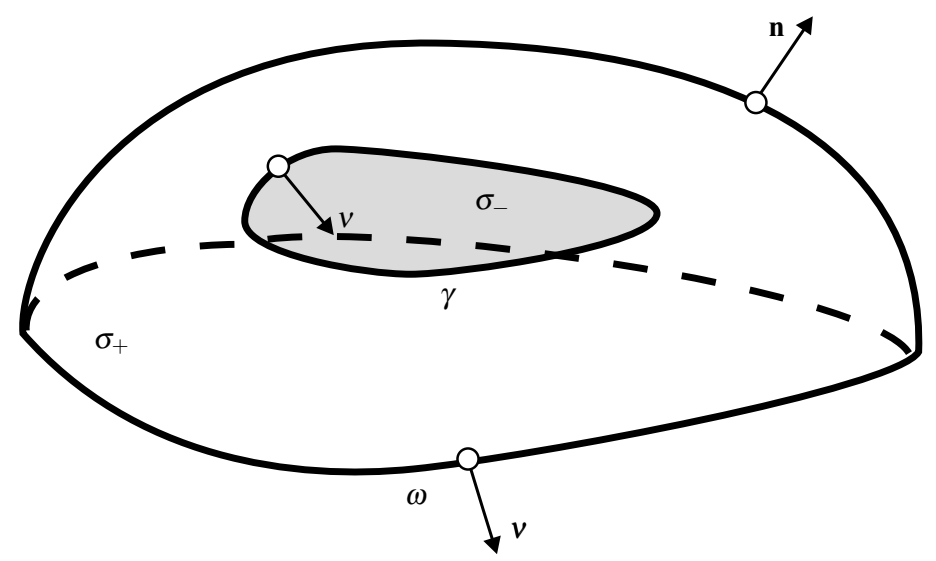

Fig. 2.3 Two-phase shell

Using the Gibbs' variational principle [28], we will find the conditions of thermodynamic equilibrium of the shell in isothermal process. For isothermal or adiabatic processes, the Gibbs' principle reduces to the stationary principle for the total energy over kinematically admissible fields of displacements and microrotations. Here we should suppose the independent position change for the border between the phases. With regard to phase transition, the variational principles of continuum mechanics are developed in [7, 29] among others.

Without the loss of generality, let us suppose that external loads are absent and that $\omega=\omega_{1}=\omega_{3}$, i.e. the displacements and rotations are given at the whole shell contour. Now the strain energy functional takes the form 


$$
\varepsilon_{1}=\mathcal{E} \equiv \iint_{\sigma} W \mathrm{~d} \sigma
$$

To find the first variation of Eq. (2.67), we use the method presented in [14]. Let us consider functional (2.67) on a smooth single-parameter family

$$
\mathbf{R}=\mathbf{R}\left(q^{\alpha}, \tau\right), \quad \mathbf{Q}=\mathbf{H}\left(q^{\alpha}, \tau\right),
$$

each element of which satisfies the border conditions for all $\tau$. Here $\tau$ is a small time-like parameter. When $\tau=0$ deformation (2.68) coincides with (2.1). We accept that in the reference configuration the shell surface as well as the border between the phases depend on $\tau$ as well

$$
\sigma=\sigma(\tau), \quad \gamma=\gamma(\tau)
$$

and $\gamma(\tau)$ lies on $\sigma(\tau)$. In other words, we will study the change of the energy functional in case of variable reference configuration. From the physical point of view, the change of the reference configuration relates to the motion of $\gamma(\tau)$ on $\sigma$ due to phase transition. We consider the boundary motion to be independent of the strain and rotation fields. This means the surfaces $\sigma$ and $\sigma(\tau)$ differ one from another only by the partition for different material phases by curves $\gamma$ and $\gamma(\tau)$, respectively.

Let $\mathbf{r}=\mathbf{r}^{*}\left(q^{\alpha}, \tau\right)$ be an arbitrary diffeomorphism mapping $\sigma$ to $\sigma(\tau)$ and such that $\gamma(\tau)=\mathbf{r}^{*}(\gamma, \tau)$. For sufficiently small $\tau$ such a map always exists. We can suppose its support to lie in some small neighborhood of $\gamma$. Then

$$
\mathcal{E}(\tau)=\iint_{\sigma(\tau)} W(\mathbf{E}(\tau), \mathbf{K}(\tau)) \mathrm{d} \sigma(\tau),
$$

where $\mathbf{E}(\tau), \mathbf{K}(\tau)$ are the strain measure and the bending strain measure, respectively, that correspond to the family (2.68). We introduce the notations

$$
\begin{gathered}
\mathbf{u}=\left.\frac{\partial \mathbf{R}\left(q^{\alpha}, \tau\right)}{\partial \tau}\right|_{\tau=0}, \quad \boldsymbol{\psi}=\left.\frac{1}{2}\left(\frac{\partial \mathbf{H}\left(q^{\alpha}, \tau\right)}{\partial \tau} \cdot \mathbf{H}^{T}\right)_{\times}\right|_{\tau=0}, \\
\mathbf{w}=\left.\frac{\partial \mathbf{r}^{*}\left(q^{\alpha}, \tau\right)}{\partial \tau}\right|_{\tau=0} .
\end{gathered}
$$

In Eqs (2.70), $\mathbf{u}, \boldsymbol{\psi}, \mathbf{w}$ are the vectors of small additional displacements, of microrotations and the vector of small perturbations of the reference configuration $\sigma$, respectively.

The first variation of $\mathcal{E}$ is the Gâteaux derivative, it can be calculated by the formula

$$
\delta \mathcal{E}=\left.\frac{\mathrm{d} \mathcal{E}}{\mathrm{d} \tau}\right|_{\tau=0} .
$$

Using integration over $\sigma$ in Eq. (2.69) and the formula of differentiation of an area element, finally we get 


$$
\delta \mathcal{E}=\iint_{\sigma}\left\{\frac{\mathrm{d} W}{\mathrm{~d} \tau}+W \nabla \cdot \mathbf{w}\right\} \mathrm{d} \sigma
$$

Here and in what follows, in the notation we will omit $\tau=0$. Vector $\mathbf{w}$ belongs to the tangent plane to $\sigma$. Indeed, $\mathbf{n} \cdot \mathbf{w}=0$ as $\mathbf{w}$ is the infinitesimal velocity vector of curve $\gamma$ that changes the position on the immovable surface $\sigma$. So we derive

$$
\delta \mathcal{E}=\iint_{\sigma}\left\{\frac{\mathrm{d} W}{\mathrm{~d} \tau}-\mathbf{w} \cdot \nabla W\right\} \mathrm{d} \sigma+\oint_{\gamma} v \cdot[[W \mathbf{w}] \mathrm{d} s,
$$

where $v$ is the normal vector to $\gamma$ that is in the tangent plane to $\sigma$. The normal direction is from $\sigma_{+}$to $\sigma_{-}$, see Fig. 2.3. Here in what follows, we denote the jump in quantities on $\gamma$ with square brackets. For example, $[[W]]=W_{+}-W_{-}$. Where it will be essential, we will use subscripts "+" and "-" of the notation of quantities for different phases. Let us denote expression $\boldsymbol{v} \cdot \mathbf{w}$ by $\mathcal{L}_{\gamma}$. Quantity $\mathcal{L}_{\gamma}$ is the virtual velocity of the motion of $\gamma$ in the direction $v$; it differs from the velocity $V$ of a singular curve in the previous section in notation only. So we get

$$
\frac{\partial \mathbf{E}}{\partial \tau}=(\nabla \mathbf{u}) \cdot \mathbf{Q}^{T}+(\mathbf{F} \times \boldsymbol{\psi}) \cdot \mathbf{Q}^{T}, \quad \frac{\partial \mathbf{K}}{\partial \tau}=(\nabla \boldsymbol{\psi}) \cdot \mathbf{Q}^{T} .
$$

Calculating the complete derivative with respect to $\tau$, we take into account that the independent variables also depend on $\tau$. Using Eqs (2.72) we can demonstrate that

$$
\frac{\mathrm{d} W}{\mathrm{~d} \tau}-\mathbf{u} \cdot \nabla W=\frac{\partial W}{\partial \mathbf{E}} \bullet \frac{\partial \mathbf{E}}{\partial \tau}+\frac{\partial W}{\partial \mathbf{K}} \bullet \frac{\partial \mathbf{K}}{\partial \tau}=\mathbf{D} \bullet \nabla \mathbf{u}+\mathbf{G} \bullet \nabla \psi-\left[\mathbf{F}^{T} \cdot \mathbf{D}\right]_{\times} \cdot \psi \cdot
$$

Thus

$$
\delta \mathcal{E}=\iint_{\sigma}\left\{\mathbf{D} \bullet \nabla \mathbf{u}+\mathbf{G} \bullet \nabla \boldsymbol{\psi}-\left[\mathbf{F}^{T} \cdot \mathbf{D}\right]_{\times} \cdot \boldsymbol{\psi}\right\} \mathrm{d} \sigma+\oint_{\gamma} \mathcal{L}_{\gamma}[[W] \mathrm{d} s,
$$

and the energy variation does not depend on the choice of diffeomorphism $\mathbf{r}^{*}$ and Eq. (2.73) includes only the motion of $\gamma$.

With regard to Eq. (2.11), we get

$$
\begin{gathered}
\delta \mathcal{E}=\delta \mathcal{J}_{\sigma}+\delta \mathcal{J}_{\gamma}, \\
-\delta \mathcal{J}_{\sigma}=\iint_{\sigma}\left\{(\boldsymbol{\nabla} \cdot \mathbf{D}) \cdot \mathbf{u}+\left[(\nabla \cdot \mathbf{G})+\left(\mathbf{F}^{T} \cdot \mathbf{D}\right)_{\times}\right] \cdot \boldsymbol{\psi}\right\} \mathrm{d} \sigma, \\
\delta \mathcal{J}_{\gamma}=\oint_{\gamma}\left\{\boldsymbol{v} \cdot[[\mathbf{D} \cdot \mathbf{u}+\mathbf{G} \cdot \boldsymbol{\psi}]]+\mathcal{L}_{\gamma}[[W]]\right\} \mathrm{d} s .
\end{gathered}
$$

Thus, the condition of stationarity

$$
\delta \mathcal{E}=0
$$


splits into two independent equations $\delta \mathcal{J}_{\sigma}=0$ and $\delta \mathcal{J}_{\gamma}=0$. From the first equation it follows the equilibrium equations (2.2.1) in each of the phase domains. To analyze what happens when the contour integral $\delta \mathcal{J}_{\gamma}=0$ takes zero value, we should introduce some assumptions on the nature of variations $\mathbf{u}$ and $\psi$ in a neighborhood of $\gamma$. The assumptions follow from the smoothness properties of $\mathbf{R}$ and $\mathbf{Q}$. Vector $\mathbf{R}$ must be continuous on $\sigma$, otherwise the shell lacks its continuity. It follows the formula that relates the jump of the additional displacement vector to the jump of the deformation gradient

$$
[[\mathbf{u}]]+\mathcal{L}_{\gamma} \nu \cdot[[\mathbf{F}]]=0 .
$$

We will differ the coherent phase interface, that is when microrotation tensor $\mathbf{Q}$ is continuous, from the phase interface incoherent in rotations, that is when its continuity on the border between phases its continuity fails. For coherent phase interface, we obtain the relation for the jumps as it follows

$$
\left[[\psi]+\mathcal{L}_{\gamma} v \cdot[[\mathbf{K} \cdot \mathbf{Q}]]=0 .\right.
$$

For the phase transition incoherent in rotations, the variations $\psi_{ \pm}$are independent and so the last relation is not valid.

Using Eqs (2.75), (2.76) we can transform equation $\delta \mathcal{J}_{\gamma}=0$ to the form

$$
\left.\oint_{\gamma}\{v \cdot[\mathbf{D}]] \cdot \mathbf{u}_{-}+v \cdot[[\mathbf{G}]] \cdot \boldsymbol{\psi}_{-}-\mathcal{L}_{\gamma} \boldsymbol{v} \cdot[[\mu]] \cdot \boldsymbol{v}\right\} \mathrm{d} s=0,
$$

where $\mathbf{C}=W \mathbf{A}-\mathbf{D} \cdot \mathbf{F}^{T}-\mathbf{G} \cdot \mathbf{Q}^{T} \cdot \mathbf{K}^{T}$ is the energy-momentum tensor or Eshelby tensor for the shell coherent phase interface [17].

For three-dimensional bodies, the Eshelby tensor describes the energy change when singularity moves inside it $[35,43]$. It describes the motion of cracks, phase interfaces, dislocations, shear bands, etc. $\mathbf{C}$ can be also represented in the following form

$$
\mathbf{C}=W \mathbf{A}-\frac{\partial W}{\partial \mathbf{E}} \cdot \mathbf{E}^{T}-\frac{\partial W}{\partial \mathbf{K}} \cdot \mathbf{K}^{T} .
$$

Within the framework of linear theory of plates and shells the properties Eshelbytype tensors are discussed in [35].

As $\mathbf{u}_{-}, \psi_{-}$and $\mathcal{L}_{\gamma}$ in Eq. (2.77) are arbitrary, by Eq. (2.77), the thermodynamic equilibrium conditions of the coherent phase interface take the form

$$
v \cdot[[\mathbf{D}]]=\mathbf{0}, \quad \boldsymbol{v} \cdot[[\mathbf{G}]]=\mathbf{0}, \quad v \cdot[[\mathbf{C}]] \cdot v=0 .
$$

The first two relations of Eqs (2.78) express the balance conditions for the forces and couples on the singular curve $\gamma$ in the equilibrium state; the last equation is an additional thermodynamic condition that is necessary for finding the position of the pre-image of $\gamma$.

It can be shown that for the phase transition incoherent in rotations, we must change the second equation in Eqs (2.78) to 


$$
v \cdot \mathbf{G}_{ \pm}=\mathbf{0} .
$$

In [17] the phase equilibrium conditions (2.78) and (2.79) are derived in another way. For the geometrically linear shell theory, the phase equilibrium conditions in displacements and rotations are formulated in [23]. Besides, in [23] they are presented in terms of stress functions.

\subsubsection{Kinetic Equation}

The above results allow us to find the time rate change of the strain energy for the micropolar shell on equilibrium deformation fields that is due to motion of singular curve $\gamma$ along the shell. It can be found for any physical phenomenon in which $\gamma$ arises. Namely, there holds

$$
\frac{\mathrm{d} \mathcal{E}}{\mathrm{d} t}=\oint_{\gamma} \mathcal{L}_{\gamma} \boldsymbol{v} \cdot[[\mathbf{C}]] \cdot \boldsymbol{v} \mathrm{d} s .
$$

In terms of linear irreversible thermodynamics,

$$
v \cdot[[\mathbf{C}]] \cdot v
$$

is the configurational force, cf. $[6,30,35])$, that is dual to $\mathcal{L}_{\gamma}$. Equation (2.80) allows us to formulate the kinetic equation to describe the motion of $\gamma$ in the surface under small perturbations from thermodynamic equilibrium

$$
\left.\mathcal{L}_{\gamma}=-\mathscr{K}(\boldsymbol{v} \cdot[\mathbf{C}]] \cdot \boldsymbol{v}\right),
$$

where $\mathscr{K}$ is a positive definite kinetic function. Equation (2.81) is analogous to the kinetic equations of the three-dimensional elasticity of two-phase solids, cf. [1,6]. Equations (2.80) and (2.81) describe the energy change for the motion of a defect in the micropolar shell. We can generalize Eq. (2.81) with regard for some additional factors such as the energy of $\gamma$, that affect its motion, see [19, 54] for details.

After $[1,6,20]$, we use the kinetic function $K(\varsigma)$ in the form

$$
\mathscr{K}(\varsigma)= \begin{cases}\frac{k\left(\varsigma-\varsigma_{0}\right)}{1+\xi\left(\varsigma-\varsigma_{0}\right)} & \varsigma \geq \varsigma_{0}, \\ 0 & -\varsigma_{0}<\varsigma<\varsigma_{0}, \\ \frac{k\left(\varsigma+\varsigma_{0}\right)}{1-\xi\left(\varsigma+\varsigma_{0}\right)} & \varsigma \leq-\varsigma_{0} .\end{cases}
$$

Here $k$ is a positive kinetic factor, $\varsigma_{0}$ describes the effects associated with nucleation of the new phase and action of the surface tension, see [1], and $\xi$ is a parameter describing the limit value of the phase interface velocity [6]. 


\subsection{Conclusions}

In this paper the basic relations of the nonlinear micropolar shell theory are reviewed. In Sect. 2.2 the local equilibrium equations and the dynamic boundary conditions of the micropolar shell are derived in terms of the surface stress and couple stress measures using the principle of virtual work. The constitutive equations for elastic shells are defined through the surface strain energy density depending on two surface strain measures by using the frame-indifference principle. Some variational principles are formulated and the nonlinear compatibility conditions for the surface strain measures are presented.

Then in Sec. 2.3 we formulate the differential form of the Coleman-Noll condition which is an analog to the GCN-condition in 3D elasticity. In 3D nonlinear elasticity the so-called constitutive restrictions or constitutive inequalities are presented in $[57,58]$. From the physical point of view these restrictions express the our ideas on the physically reasonable behavior of materials. In the linear shell theory, such a restriction is given by the condition of positive definiteness of the shell strain energy. For finite deformation, definite positiveness of the energy is not sufficient. In Sect. 2.3 we also consider the strong ellipticity condition, the Coleman-Noll inequality and the condition of ordinary ellipticity. Then we deduce the linearized equilibrium equations and formulate the strong ellipticity condition (2.55) and the Hadamard inequality. We proved that the Coleman-Noll condition is more general and it implies the strong ellipticity of the equilibrium shell equations. We also show that the strong ellipticity condition is equivalent to the conditions of the existence of accelerations waves in the shell. We establish that the conditions of ordinary ellipticity are more weak. When they fail then there exist non-smooth solutions of the shell equilibrium equations.

In Sect. 2.4 we establish the conditions of thermodynamic equilibrium for shells undergoing phase transitions. In case of small deviation from thermodynamic equilibrium, we formulate a kinetic equation that describe the motion of the phase interface.

Acknowledgements The second author was supported by the DFG grant No. AL 341/33-1 and by the RFBR with the grant No. 09-01-00459.

\section{References}

[1] Abeyaratne, R., Knowles, J.K.: Evolution of Phase Transitions. A Continuum Theory. Cambridge University Press, Cambridge (2006)

[2] Agrawal, A., Steigmann, D.J.: Coexistent fluid-phase equilibria in biomembranes with bending elasticity. Journal of Elasticity 93(1), 63-80 (2008)

[3] Altenbach, H., Eremeyev, V.A., Lebedev, L.P., Rendón, L.A.: Acceleration waves and ellipticity in thermoelastic micropolar media. Arch. Appl. Mech. 80(3), 217-227 (2010) 
[4] Altenbach, H., Zhilin, P.A.: A general theory of elastic simple shells (in Russian). Uspekhi Mekhaniki (Advances in Mechanics) 11(4), 107-148 (1988)

[5] Altenbach, J., Altenbach, H., Eremeyev, V.A.: On generalized Cosserat-type theories of plates and shells. A short review and bibliography. Arch. Appl. Mech. 80(1), 73-92 (2010)

[6] Berezovski, A., Engelbrecht, J., Maugin, G.A.: Numerical Simulation of Waves and Fronts in Inhomogeneous Solids. World Scientific, New Jersey et al. (2008)

[7] Bhattacharya, K.: Microstructure of Martensite: Why It Forms and How It Gives Rise to the Shape-Memory Effect. Oxford University Press, Oxford (2003)

[8] Bhattacharya, K., James, R.D.: A theory of thin films of martensitic materials with applications to microactuators. J. Mech. Phys. Solids 36, 531-576 (1999)

[9] Boulbitch, A.A.: Equations of heterophase equilibrium of a biomembrane. Archive of Applied Mechanics 69(2), 83-93 (1999)

[10] Chróścielewski, J., Makowski, J., Pietraszkiewicz, W.: Statics and Dynamics of Multyfolded Shells. Nonlinear Theory and Finite Elelement Method. Wydawnictwo IPPT PAN, Warszawa (2004)

[11] Chróścielewski, J., Pietraszkiewicz, W., Witkowski, W.: On shear correction factors in the non-linear theory of elastic shells. Int. J. Solids Struct. 47(2526), 3537-3545 (2010)

[12] Chróścielewski, J., Witkowski, W.: On some constitutive equations for micropolar plates. ZAMM 90(1), 53-64 (2010)

[13] Cosserat, E., Cosserat, F.: Théorie des Corps Déformables. Hermann Editeurs, Paris (1909) (Reprint, Gabay, Paris, 2008)

[14] Courant, R., Hilbert, D.: Methods of Mathematical Physics, Vol. 1. Wiley, New York (1991)

[15] Eremeyev, V.A.: Acceleration waves in micropolar elastic media. Doklady Physics 50(4), 204-206 (2005)

[16] Eremeyev, V.A.: Nonlinear micropolar shells: theory and applications. In: W. Pietraszkiewicz, C. Szymczak (eds.) Shell Structures: Theory and Applications, pp. 11-18. Taylor \& Francis, London (2005)

[17] Eremeyev, V.A., Pietraszkiewicz, W.: The non-linear theory of elastic shells with phase transitions. J. Elasticity 74(1), 67-86 (2004)

[18] Eremeyev, V.A., Pietraszkiewicz, W.: Local symmetry group in the general theory of elastic shells. J. Elasticity 85(2), 125-152 (2006)

[19] Eremeyev, V.A., Pietraszkiewicz, W.: Phase transitions in thermoelastic and thermoviscoelastic shells. Arch. Mech. 61(1), 41-67 (2009)

[20] Eremeyev, V.A., Pietraszkiewicz, W.: On tension of a two-phase elastic tube. In: W. Pietraszkiewicz, I. Kreja (eds.) Shell Structures. Theory and Applications. Vol. 2., pp. 63-66. CRC Press, Boca Raton (2010)

[21] Eremeyev, V.A., Zubov, L.M.: On the stability of elastic bodies with couple stresses (in Russian). Izv. RAN. Mekanika Tvedogo Tela (Mechanics of Solids) (3), 181-190 (1994) 
[22] Eremeyev, V.A., Zubov, L.M.: On constitutive inequalities in nonlinear theory of elastic shells. ZAMM 87(2), 94-101 (2007)

[23] Eremeyev, V.A., Zubov, L.M.: Mechanics of Elastic Shells (in Russian). Nauka, Moscow (2008)

[24] Ericksen, J.L., Truesdell, C.: Exact theory of stress and strain in rods and shells. Arch. Rat. Mech. Analysis 1(1), 295-323 (1958)

[25] Eringen, A.C.: Microcontinuum Field Theory. I. Foundations and Solids. Springer, New York (1999)

[26] Eringen, A.C.: Microcontinuum Field Theory. II. Fluent Media. Springer, New York (2001)

[27] Fichera, G.: Existence theorems in elasticity. In: S. Flügge (ed.) Handbuch der Physik, vol. VIa/2, pp. 347-389. Springer, Berlin (1972)

[28] Gibbs, J.W.: On the equilibrium of heterogeneous substances. In: The Collected Works of J. Willard Gibbs, pp. 55-353. Longmans, Green \& Co, New York (1928)

[29] Grinfeld, M.: Thermodynamics Methods in the Theory of Heterogeneous Systems. Longman, Harlow (1991)

[30] Gurtin, M.E.: Configurational Forces as Basic Concepts of Continuum Physics. Springer-Verlag, Berlin (2000)

[31] He, Y.J., Sun, Q.: Scaling relationship on macroscopic helical domains in NiTi tubes. Int. J. Solids Struct. 46(24), 4242-4251 (2009)

[32] He, Y.J., Sun, Q.: Macroscopic equilibrium domain structure and geometric compatibility in elastic phase transition of thin plates. Int. J. Mech. Sci. 52(2), 198-211 (2010)

[33] He, Y.J., Sun, Q.: Rate-dependent domain spacing in a stretched NiTi strip. Int. J. Solids Struct. 47(20), 2775-2783 (2010)

[34] James, R.D., Rizzoni, R.: Pressurized shape memory thin films. J. Elasticity 59, 399-436 (2000)

[35] Kienzler, R., Herrman, G.: Mechanics in Material Space with Applications to Defect and Fracure Mechanics. Springer-Verlag, Berlin (2000)

[36] Lebedev, L.P., Cloud, M.J., Eremeyev, V.A.: Tensor Analysis with Applications in Mechanics. World Scientific, New Jersey (2010)

[37] Li, Z.Q., Sun, Q.: The initiation and growth of macroscopic martensite band in nano-grained NiTi microtube under tension. Int. J. Plasticity 18(11), 1481$1498(2002)$

[38] Libai, A., Simmonds, J.G.: Nonlinear elastic shell theory. Adv. Appl. Mech. 23, 271-371 (1983)

[39] Libai, A., Simmonds, J.G.: The Nonlinear Theory of Elastic Shells, 2nd edn. Cambridge University Press, Cambridge (1998)

[40] Lions, J.L., Magenes, E.: Problèmes aux limites non homogènes et applications. Dunod, Paris (1968)

[41] Lurie, A.I.: Analytical Mechanics. Foundations of Engineering Mechanics. Springer, Berlin (2001)

[42] Lurie, A.I.: Theory of Elasticity. Foundations of Engineering Mechanics. Springer, Berlin (2005) 
[43] Maugin, G.A.: Material Inhomogeneities in Elasticity. Chapman Hall, London (1993)

[44] Maugin, G.A.: A historical perspective of generalized continuum mechanics. In: H. Altenbach, V.I. Erofeev, G.A. Maugin (eds.) Mechanics of Generalized Continua. From the Micromechanical Basics to Engineering Applications. Springer, Berlin (2011)

[45] Maugin, G.A., Metrikine, A.V. (eds.): Mechanics of Generalized Continua: One hundred years after the Cosserats. Springer, New York (2010)

[46] Miyazaki, S., Fu, Y.Q., Huang, W.M. (eds.): Thin Film Shape Memory Alloys: Fundamentals and Device Applications. Cambridge University Press, Cambridge (2009)

[47] Nowacki, W.: Theory of Asymmetric Elasticity. Pergamon-Press, Oxford et al. (1986)

[48] Pietraszkiewicz, W.: Consistent second approximation to the elastic strain energy of a shell. ZAMM 59, 206-208 (1979)

[49] Pietraszkiewicz, W.: Finite Rotations and Langrangian Description in the Non-linear Theory of Shells. Polish Sci. Publ, Warszawa-Poznań (1979)

[50] Pietraszkiewicz, W.: Geometrically nonlinear theories of thin elastic shells. Uspekhi Mechaniki (Advances in Mechanics) 12(1), 51-130 (1989)

[51] Pietraszkiewicz, W.: Teorie nieliniowe powłok. In: C. Woźniak (ed.) Mechanika sprężystych płyt i powłok, pp. 424-497. PWN, Warszawa (2001)

[52] Pietraszkiewicz, W., Eremeyev, V.A.: On natural strain measures of the nonlinear micropolar continuum. Int. J. Solids Struct. 46, 774-787 (2009)

[53] Pietraszkiewicz, W., Eremeyev, V.A.: On vectorially parameterized natural strain measures of the non-linear Cosserat continuum. Int. J. Solids Struct. 46(11-12), 2477-2480 (2009)

[54] Pietraszkiewicz, W., Eremeyev, V.A., Konopińska, V.: Extended non-linear relations of elastic shells undergoing phase transitions. ZAMM 87(2), 150-159 (2007)

[55] Reissner, E.: Linear and nonlinear theory of shells. In: Y.C. Fung, E.E. Sechler (eds.) Thin Shell Structures, pp. 29-44. Prentice-Hall, Englewood Cliffs, NJ (1974)

[56] Truesdell, C.: Rational Thermodynamics, 2nd edn. Springer, New York (1984)

[57] Truesdell, C.: A First Course in Rational Continuum Mechanics, 2nd edn. Academic Press, New York (1991)

[58] Truesdell, C., Noll, W.: The nonlinear field theories of mechanics. In: S. Flügge (ed.) Handbuch der Physik, Vol. III/3, pp. 1-602. Springer, Berlin (1965)

[59] Zhilin, P.A.: Mechanics of deformable directed surfaces. Int. J. Solids Struct. 12, $635-648$ (1976)

[60] Zubov, L.M.: Nonlinear Theory of Dislocations and Disclinations in Elastic Bodies. Springer, Berlin (1997) 